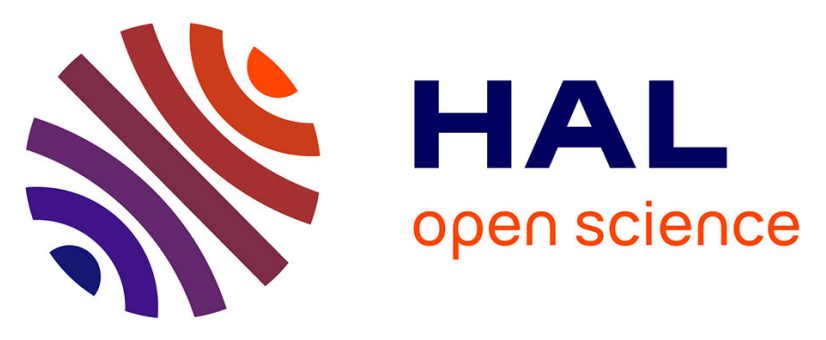

\title{
125Te NMR for structural investigations in phase change materials: Optimization of experimental conditions coupled to NMR shift prediction
}

Juliette Lizion, Andrea Piarristeguy, Robert Laskowski, Peter Blaha, Raphael Escalier, Michel Ménétrier, Annie Pradel, Gilles Silly

\section{To cite this version:}

Juliette Lizion, Andrea Piarristeguy, Robert Laskowski, Peter Blaha, Raphael Escalier, et al.. 125Te NMR for structural investigations in phase change materials: Optimization of experimental conditions coupled to NMR shift prediction. Solid State Nuclear Magnetic Resonance, 2021, 115, pp.101751. 10.1016/j.ssnmr.2021.101751 . hal-03318557

\section{HAL Id: hal-03318557 https://hal.science/hal-03318557}

Submitted on 10 Aug 2021

HAL is a multi-disciplinary open access archive for the deposit and dissemination of scientific research documents, whether they are published or not. The documents may come from teaching and research institutions in France or abroad, or from public or private research centers.
L'archive ouverte pluridisciplinaire HAL, est destinée au dépôt et à la diffusion de documents scientifiques de niveau recherche, publiés ou non, émanant des établissements d'enseignement et de recherche français ou étrangers, des laboratoires publics ou privés. 
${ }^{125}$ Te NMR for structural investigations in Phase Change Materials: optimization of experimental conditions coupled to NMR shift prediction

J. Lizion ${ }^{1}$, A. Piarristeguy ${ }^{1}$, R. Laskowsky ${ }^{2}$, P. Blaha ${ }^{3}$, R. Escalier ${ }^{1}$, M. Ménétrier ${ }^{4}$, A. Pradel ${ }^{1}$, G. Silly ${ }^{1}$

1-ICGM, Univ Montpellier, CNRS, ENSCM, Montpellier, France.

2-Institute of High Performance Computing, A*STAR, 1 Fusionopolis Way, \#16-16, Connexis, Singapore 138632

3-Institute of Materials Chemistry, Vienna University of Technology, Getreidemarkt 9/165-TC, A-1060 Vienna, Austria

4-ICMCB-CNRS, Univ. Bordeaux, Bordeaux INP, ICMCB UMR 5026, F-33600 Pessac, France

\begin{abstract}
Phase Change Materials as those of the Ge-Sb-Te ternary system are of great interest for technological applications. Properties of these compounds are strongly related to presence of vacancies and structural investigations remain challenging. In this paper we evidence that ${ }^{125} \mathrm{Te}$ NMR in natural abundance and using commercial systems at intermediate field (14.1T) together with NMR parameters prediction can contribute to improve understanding of electronic structure of such systems. GeTe is a typical phase change material, whose structure contains germanium vacancies, even in its stoichiometric form, giving it metallic properties. Here, we use nominal $\mathrm{Ge}_{50} \mathrm{Te}_{50}$ and $\mathrm{Ge}_{48} \mathrm{Te}_{52}$ crystalline samples as an example to optimize the WURST-CPMG technique, a powerful technique to record wide NMR spectra which has not yet been used on ${ }^{125} \mathrm{Te}$. The goal was to minimize the time devoted to experiments as well as maximize the signal-to-noise ratio in order to detect small intensity signals directly linked to vacancies. Virtual Crystal Approximation (VCA) calculations performed with WIEN2K helped to interpret the NMR spectra. For Te-based crystalline conducting samples the best experimental results were obtained using $3.2 \mathrm{~mm}$ thin wall rotors with diluted samples 40 vol.\% GeTe-60 vol.\% $\mathrm{SiO}_{2}$. In addition to the WURST-CPMG technique, high resolution spectra using MAS as implemented in the pj-MAT technique allowed us to identify the distributions of
\end{abstract}


chemical shift parameters in the high intensity contribution of the 1D spectra. The NMR spectra recorded on the samples showed that an addition of Tellurium in the stoichiometric $\mathrm{Ge}_{50} \mathrm{Te}_{50}$ sample leads to an important broadening of the spectrum together with a shift of the lines. According to VCA calculations it could be attributed to a distribution of concentrations of germanium vacancies in the sample and it would appear that Knight Shift but also Chemical Shift could contribute in similar proportion to the NMR line position when metavalent bonding is invoked.

\section{Keywords}

Solid State NMR, Phase Change Materials, Chemical Shift, Knight Shift, vacancies, WURST-CPMG, pjMAT

\section{Introduction}

Over the past two decades, Phase change Materials (PCM) have received a huge attention with various technological applications ranging from optical discs, such as CD-RW or DVD-RW discs to phase-change memory (PC-RAM), including more recently opportunities as all-photonic memories and flexible displays with nanopixel resolution [1].

The Phase-Change Random Access Memories (PC-RAM), susceptible to replace the current flash memories [2], take advantage of properties of telluride chalcogenide films, i.e. their low glass transition temperature, their large differences in structure between the crystalline and amorphous phases, their low band gap, their stability of the crystalline and amorphous phases, their different optical and electrical properties, and, finally, their fast recrystallization of the amorphous phase $[1,3]$. When a voltage is applied to a device in the high-resistivity amorphous (RESET) state, it switches to the low-resistivity crystalline (SET) state.

Initially, phase change materials were optimized employing empirical schemes and a trial-and-error approach. The three most important classes of phase-change materials discovered are found on the pseudobinary tie line between $\mathrm{GeTe}$ and $\mathrm{Sb}_{2} \mathrm{Te}_{3}$, the region around $\mathrm{Sb}_{2} \mathrm{Te}$ as well as the area around $\mathrm{Sb}$. About ten years ago, however, a unique fingerprint of phase-change materials was identified [4]. All phase change 
materials displayed very large electronic polarizabilities in the crystalline state, which were not found in the amorphous phase. Therefore, the high values of the electronic polarizability of PCMs were attributed to the "resonant bonding" model. More recently other particular characteristics of these materials were discovered: moderate electronic conductivity, increased coordination numbers incompatible with the (8-N) rule for semiconductors, and large lattice anharmonicity [5]. These properties, along with large dielectric constants, were attributed to a new type of bonding called "metavalent bonding" in the crystalline state. Investigations indicate that the findings of this special bonding is a genuine mechanism in solids and not merely an intermediate (or a combination) of covalent and metallic bonding [5].

In order to explain the operation of this memory and in particular to better understand the phenomena at the origin of the switching from a resistive state to a conductive state in PC-RAM memories, the electrical, structural, and thermal properties of Ge-Sb-Te (GST) thin films have been widely studied [6][7][8]. In particular, the crystalline states obtained in phase-change devices are highly disordered, in terms of compositional randomness and structural distortion. It was shown that the role of Ge vacancies and distortions in the crystalline phase has a significant impact for the data-storage performance.

Insight into the structure of these crystalline materials was clearly needed. Until now, PCM have mainly been analyzed using neutron or/and synchrotron radiation (diffraction, EXAFS, XANES, ...) and electron microscopy (TEM, HAADF-STEM...) techniques to describe the short-range order.

NMR also allows to investigate the local order around a considered nucleus, and we focus here on the ${ }^{125} \mathrm{Te}$ nucleus which has not been studied so much in PCM up to now. On the NMR spectra the position of the line is related to the response of the electronic cloud to the application of an external static magnetic field $\mathrm{B}_{0}$. The first contribution present in any system is the Chemical Shift and corresponds to the orbital part. Hence if for instance Ge vacancies are present around tellurium atoms the chemical bond will be affected and the NMR shift will be modified compared to the standard line position without vacancy. In addition, another contribution may also have an important effect on the line position. Defects such as vacancies modify the electronic band structure of the crystalline system which can switch from insulator to metallic, this is particularly true in GST which present a low band gap. Consequently, the Knight Shift or spin 
contribution of delocalized electrons to the line position may be very large. The line position including both contributions is given using the chemical shift scale $\delta$ using a sample of reference:

$$
\delta(p p m)=\frac{v-v_{r e f}}{v_{r e f}} \cdot 10^{6}
$$

where $v$ and $v_{\text {ref }}$ are the line position in Hertz in the sample and in the reference sample respectively.

NMR is thus a technique of choice to investigate local order and the role of vacancies in PCM materials as well as metavalent bonding.

However, ${ }^{125} \mathrm{Te}$ is a non-straightforward nucleus. Its low natural abundance $(7.14 \%)$ leads to small sensitivity. Literature on experimental ${ }^{125} \mathrm{Te}$ NMR of crystalline phases is not substantial. Some data are available for some tellurium compounds [9], crystalline tellurites and tellurates [10,11] and transition-metal ditellurides [12], which represents a variety of chemical bonding for Tellurium from semiconductors to metallic systems with a chemical shift range of thousands of ppm. Then combined to local disorder due to crystalline defects, extremely large NMR linewidths are usually obtained in most of the PCM which strongly decrease signal to noise ratio $(\mathrm{S} / \mathrm{N})$. So, in order to make intense usage of this technique it is important to use efficient methods which allow to record spectra ranging on several thousands of ppm with good $\mathrm{S} / \mathrm{N}$ and not being too much time consuming.

Dealing with PCM, the first ${ }^{125} \mathrm{Te}$ NMR studies of crystalline and amorphous chalcogenides in the Ge-SbTe system were realized by Bobela and Taylor in 2008 [13][14]. The standard 90-180 Hahn echo pulse sequence was used under static conditions. As the spectra were much broader than the excitation profile of a single pulse of radio frequency energy their spectra were acquired piecewise in discrete frequency steps equal to the frequency coverage of the second (longest) pulse. Only one data point is acquired for each frequency step. Typically, 10 to 20 points are registered on a chemical shift range of $3000 \mathrm{ppm}$ for each spectrum leading to spectra with low resolution. Crystalline $\mathrm{Sb}_{2} \mathrm{Te}_{3}, \mathrm{GeTe}, \mathrm{Ge}_{2} \mathrm{Sb}_{2} \mathrm{Te}_{5}$ and amorphous $\mathrm{Ge}_{2} \mathrm{Sb}_{2} \mathrm{Te}_{4}, \mathrm{Ge}_{2} \mathrm{Sb}_{2} \mathrm{Te}_{5}$ and $\mathrm{Ge}_{2} \mathrm{Sb}_{2} \mathrm{Te}_{7}$ were investigated.

In 2011, new investigations were realized by Edwards et al. [15] in the Ge-As-Sb-Te crystallized and amorphous systems using Magic Angle Spinning (MAS) and Hahn echo pulse sequence. When necessary, to circumvent the problem of broad line shapes a frequency-sweep technique was employed, also called 
Variable-Offset-Cumulative Spectroscopy (VOCS) [16]. The central frequency was swept over $1 \mathrm{MHz}$ in increments of $0.1 \mathrm{MHz}$ in order to ensure a uniform radiofrequency excitation profile and the collected lineshapes were subsequently co-added to produce the final spectrum. In particular, crystalline GeTe and $\mathrm{Sb}_{2} \mathrm{Te}_{3}$ were reinvestigated together with crystalline fcc and hexagonal $\mathrm{Ge}_{1} \mathrm{Sb}_{2} \mathrm{Te}_{4}$.

A similar method was also used by Sen et al [17] to study amorphous and fcc $\mathrm{Ge}_{1} \mathrm{Sb}_{2} \mathrm{Te}_{4}$ and $\mathrm{Ge}_{2} \mathrm{Sb}_{2} \mathrm{Te}_{5}$, by Levin et al. and Cui et al. [18][19][20][21][22][23][24] for GeTe based materials and $\mathrm{Bi}_{2} \mathrm{Te}_{3}$ and by Njegic et al. [25] for the PbTe-GeTe binary and PbTe-SnTe binary. It was also used by Garaga et al. [26] on Te oxides but using a CPMG sequence which allows to obtain a better sensitivity.

This VOCS technique allows to obtain a better sensitivity but is still very time consuming because it requires to acquire many spectra and execute tuning and matching of the probe between each acquisition. 1D experiments are usually preferred in a first approach. Dealing with the problematic of 1D wide spectra, recent experimental methods such as WURST-CPMG pulse sequence, for static samples, allow recording a spectrum over more than a $1 \mathrm{MHz}$ range in one shot and in reasonable delays. This technique combines amplitude and phase modulated pulses allowing frequency sweeping during the pulses with CPMG technique based on echoes which allow to record few tens of free induction decay signals (instead of one) in a single scan. This technique has been developed originally on ${ }^{71} \mathrm{Ga}$ and ${ }^{91} \mathrm{Sr}$ NMR [27] but is now extensively used: more than 300 publications report the use of WURST-CPMG on a wide variety of nuclei. ${ }^{125}$ Te belongs to the family of heavy atoms which usually give wide NMR spectra. WURST-CPMG has been used in particular for such heavy nuclei [28], but never on ${ }^{125} \mathrm{Te}$.

More recent 2D (MAT-PASS and pj-MAT) techniques that are obtained using MAS allow obtaining high resolution 2D map in crystallized and disordered systems. This technique is employed to separate the Chemical Shift Anisotropy (CSA) in the dimension F2 from the isotropic chemical shift in the second F1 dimension, resulting in increased spectral resolution while preserving the information contained in the CSA. It has already been used in ${ }^{125} \mathrm{Te} \mathrm{NMR}$ in crystalline $\mathrm{As}_{2} \mathrm{Te}_{3}$ [29] and glasses: As-Te [29], $\mathrm{BaO}^{-\mathrm{TeO}_{2}}$ [30] and $\mathrm{TeO}_{2}$ [31]. However, it has not yet been used on Phase Change Materials. These studies have been realized at very high field $(18.8 \mathrm{~T})$ using a homemade probe when we are here interested in experiments that can be carried out on more standard magnets and commercial probes. 
In this paper ${ }^{125} \mathrm{Te}$ NMR in crystalline stoichiometric $\mathrm{GeTe}\left(\mathrm{Ge}_{50} \mathrm{Te}_{50}\right)$ and $\mathrm{GeTe}$ with an excess of tellurium $\left(\mathrm{Ge}_{48} \mathrm{Te}_{52}\right)$ will be reinvestigated using a WURST-CPMG pulse sequence and the pj-MAT technique. GeTe is one of the first PCM discovered, it has been studied extensively and has a relatively simple distorted rocksalt structure. The ideal GeTe is a semi-conductor, however several studies have shown that GeTe rarely exists without Ge vacancies and has a metallic behavior $[32,33,34,35,36]$. Two phases, $\alpha-\mathrm{GeTe}$ and $\gamma$-GeTe exist depending on the composition of the sample. It has already been shown experimentally that a tellurium excess in GeTe increases the vacancy concentration and broadens the NMR signal considerably [21]. Therefore, this compound is a good model for the optimization of the WURST-CPMG technique, which is suitable for the recording of very wide NMR spectra, using the ${ }^{125}$ Te nucleus. We will show how this technique has allowed us to detect signals of weak intensity in a reasonable amount of time, and how the pj-MAT technique has been used complementarily to obtain more information on the high intensity contributions of the 1D WURST-CPMG spectra.

We have also performed simple $a b$ initio calculations in WIEN2K using the Virtual Crystal Approximation (VCA) method that confirms that an important amount of vacancies can shift the GeTe ${ }^{125} \mathrm{Te}$ NMR Shift by several thousand ppm.

\section{Experimental}

\subsection{Synthesis and characterization}

Crystalline Ge-Te samples were studied by ${ }^{125} \mathrm{Te}$ NMR measurements. A stoichiometric GeTe sample $\left(\mathrm{Ge}_{50} \mathrm{Te}_{50}\right)$ and a GeTe sample with a 4 at.\% Te excess $\left(\mathrm{Ge}_{48} \mathrm{Te}_{52}\right)$ were synthesized by classical synthesis route from high-purity germanium (Goodfellow-99.999\%) and tellurium (5N Plus-99.9999\%). Around 1g of powders were introduced in a cylindrical silica tube $(8 \mathrm{~mm}$ inner diameter, $1 \mathrm{~mm}$ thick) further sealed under secondary vacuum $\left(10^{-5} \mathrm{mbar}\right)$. The ampoule containing Ge-Te powders was heated at a low heating rate of $10^{\circ} \mathrm{C} / \mathrm{h}$ until around $950^{\circ} \mathrm{C}$, using a rocking furnace to form a homogeneous melt. After 4 hours at $950^{\circ} \mathrm{C}$, the rocking was stopped, and the tubes were cooled down to room temperature at a rate of $10^{\circ} \mathrm{C} / \mathrm{h}$. 
The X-ray diffraction pattern of the crystalline sample was recorded using a PANalytical XPERT diffractometer operating in Brag-Brentano geometry $\Theta-2 \Theta$ with $\lambda_{\mathrm{Cu}}\left(\mathrm{K}_{\alpha 1}=1.5405 \AA\right.$ and $\left.\mathrm{K}_{\alpha 2}=1.5443 \AA\right)$.

\section{2. ${ }^{125}$ Te NMR measurements}

Static Solid State NMR (SSNMR) spectra where recorded on a Varian VNMRS 600 spectrometer with a magnetic field strength of 14.1 T. T3 triple resonance MAS probes HXY with Vespel stators and Zirconia 3.2 and $1.6 \mathrm{~mm}$ o.d. pencil MAS rotors were used together with a HFXY with a KelF stator and $2.5 \mathrm{~mm}$ o.d. pencil MAS rotors. We used thin wall $(36 \mu \mathrm{L})$ and standard $(22 \mu \mathrm{L}) 3.2 \mathrm{~mm}$ rotors, and $2.5 \mathrm{~mm}(11 \mu \mathrm{L})$ and $1.6 \mathrm{~mm}(8 \mu \mathrm{L})$ rotors. All the experiments were performed at room temperature without spinning for 1D spectra. The relaxation delay was set to $1 \mathrm{~s}$ which is enough for full relaxation for conductive crystallized samples.

Variable-Offset-Cumulative Spectroscopy (VOCS) [16] ${ }^{125} \mathrm{Te}$ NMR spectra were collected by moving the frequency offset in steps of $80 \mathrm{kHz}, 3600$ transients were collected so that each acquisition lasted one hour. The full shifted echoes were registered using a Hahn echo pulse sequence with a pulse duration of $2 \mu$ s for the $90^{\circ}$ pulse and a delay of $100 \mu$ s for the echo. The final spectrum was produced by adding all the 11 individual magnitude spectra.

One experiment for each sample was also performed on a VNMRS 400 spectrometer with a magnetic field of 9.4T with a T3 triple $3.2 \mathrm{~mm}$ o.d. pencil MAS rotor.

In WURST-CPMG experiments on both $14.1 \mathrm{~T}$ and 9.4T spectrometers the spectral width was $1.25 \mathrm{MHz}$. We followed the experimental methodology presented by Hung and Gan [37] using a pulse sequence presented in Figure 1.a with a two steps $\phi_{1}=0, \pi$ phase cycling of excitation pulse and no phase cycling for refocusing pulses $\left(\phi_{2}=0\right)$. The pulse durations were set to $\mathrm{P}_{1}=\mathrm{P}_{2}=50 \mu \mathrm{s}$, the frequency sweep rate was $\mathrm{R}=25 \mathrm{GHz} . \mathrm{s}^{-1}$ and the power of the two identical pulses was optimized for maximum signal. Acquisition duration was set to $\tau_{4}=100 \mu$ s. The synchronization of the pulse sequence to obtain the echo in the center of the acquisition windows was set using $\tau_{1}=20.6 \mu \mathrm{s}$ and dead time $\tau_{2}=\tau_{3}=5.2 \mu$ s corresponding to maximum intensity on the spectra. $\mathrm{N}=163$ echoes were registered. The spectra were recorded in $\sim 12 \mathrm{~h}$. 
One spectrum was recorded at lower field on an AVANCE BRUKER spectrometer with a magnetic field of 2.35T and a 2.5mm o.d. MAS rotor. The sample was not from the same batch as for other experiments but prepared in the same way. The experiment was realized using standard CPMG technic with a $1.4 \mu \mathrm{s} 90^{\circ}$ and a $2.8 \mu \mathrm{s} 180^{\circ}$ pulse duration, 100 echoes were registered with an echo delay of $131 \mu \mathrm{s}$ and $1 \mathrm{~s}$ recycle delay. Spinning rate was $30 \mathrm{kHz}$.

The 2D pjMAT/CPMG spectra were acquired using the pulse sequence described by Kaseman et al. [29] presented in Figure 1.b) with $a=1, b=4$. The spinning rate was $10 \mathrm{kHz}$ leading to $\tau_{\mathrm{R}}=100 \mu \mathrm{s}$. The dwell time in $\mathrm{F} 1$ dimension is rotor synchronized with $\delta \mathrm{t}_{1}=\tau_{\mathrm{R}}, \tau_{\mathrm{E}}=200 \mu \mathrm{s} .32$ hypercomplex $\mathrm{t}_{1}$ data points were used, with 1440 transients per point,130 echoes per transient and a recycle delay of $1 \mathrm{~s}$. The spectral width was $320 \mathrm{kHz}$ in the F1 dimension and 1.25 MHz in the F2 dimension. The spectra were acquired in $\sim 24 \mathrm{~h}$, with a $3 \mu \mathrm{s} 90^{\circ}$ and a $6 \mu \mathrm{s} 180^{\circ}$ pulse duration. For $\mathrm{Ge}_{48} \mathrm{Te}_{52}$ three spectra were recorded, with three different offsets in order to recover the entirety of the signal. Only two of those spectra are presented, because the third one is only noise.

The conductivity of Ge-Te crystalline samples is sufficiently large (around $5.10^{4} \mathrm{~S} / \mathrm{cm}$ ) to make impossible tuning and matching of the probe. To overcome this problem, we mixed the samples with a fine grounded $\mathrm{SiO}_{2}$ powder to be able to set up the measurement. The powders were crushed in an agate mortar and sieved through a $125 \mu \mathrm{m}$ mesh stainless steel sieve before mixing. We prepared several NMR samples of $\mathrm{Ge}_{48} \mathrm{Te}_{52}$ with different $\mathrm{SiO}_{2}$ percentages by volume (from $20 \%$ to $80 \%$ ) in order to determine the one that produces the best signal to noise ratio $(\mathrm{S} / \mathrm{N})$. The optimization of $\mathrm{SiO}_{2}$ percentage and $\mathrm{NMR}$ parameters were carried out on $\mathrm{Ge}_{48} \mathrm{Te}_{52}$ because it contains more vacancies than $\mathrm{Ge}_{50} \mathrm{Te}_{50}$ and thus has a higher conductivity. The optimized parameters were then used for $\mathrm{Ge}_{50} \mathrm{Te}_{50}$.

The isotropic chemical shift $\delta_{\text {iso }}$ was defined as $\delta_{\text {iso }}=\frac{1}{3}\left(\delta_{\mathrm{xx}}+\delta_{\mathrm{yy}}+\delta_{\mathrm{zz}}\right)$ with $\delta_{\mathrm{ii}}=\sigma_{\text {ref }}-\sigma_{\mathrm{ii}}$ and $\sigma_{\mathrm{ii}}(\mathrm{i}=\mathrm{x}$, $\mathrm{y}, \mathrm{z})$ the principal components of the shielding tensor. The anisotropy $\Delta_{\mathrm{CS}}$ is defined as $\Delta_{\mathrm{CS}}=3 / 2 \delta^{*}$ with $\delta^{*}=\delta_{\mathrm{zz}}-\delta_{\text {iso. }}$. The asymmetry parameter $\eta$ is defined as $\eta=\left(\delta_{\mathrm{yy}}-\delta_{\mathrm{xx}}\right) / \delta^{*}$. In the case of an axial symmetry where $\delta_{\mathrm{xx}}=\delta_{\mathrm{yy}}=\delta_{\perp}$ and $\delta_{\mathrm{zz}}=\delta_{/ /,}, \eta=0 . \mathrm{ZnTe}$ was used as a secondary reference at $-888 \mathrm{ppm}$ with a principal reference $\left(\mathrm{CH}_{3}\right)_{2} \mathrm{Te}$ at $0 \mathrm{ppm}$. 


\section{3. ${ }^{125}$ Te NMR shift calculations}

${ }^{125} \mathrm{Te}$ NMR shifts calculations of the Ge-Te structures with vacancies were performed using WIEN2K package [38] using the Virtual Crystal Approximation (VCA) with the PBE-GGA exchange-correlation functional. The calculations are performed on the perfect structure without vacancies, but the number of electrons in the cell is modified to simulate the effect on the electronic density of a certain mean concentration of vacancies. For $\alpha-\mathrm{GeTe}$ and $\gamma$-GeTe the lattice parameters and atomic positions were obtained from [39] and [40] respectively. In order to simulate effect of vacancies, the number of valence electrons in the cell was reduced from 30 in the perfect structure to 29.8 by GeTe unit. This allowed to vary the charge carrier concentration from 0 to $3.510^{21} \mathrm{~cm}^{-3}$.

The value of the cutoff parameter RKmax that controls the magnitude of the largest $\mathrm{K}$ vector was set to 8; 512000 and $16000 \mathrm{k}$-points were taken for $\alpha$-GeTe and $\gamma$-GeTe respectively in the self-consistent calculations for the orbital part, and $128000 \mathrm{k}$-points were taken for both structures for the spin part.

The isotropic screen tensor value corresponding to the orbital contribution and the spin contribution are added to obtain the total isotropic screening coefficient $\sigma_{\text {iso }}: \sigma_{\text {iso-orbital }}+\sigma_{\text {spin }}=\sigma_{\text {iso. }} \sigma_{\text {iso-orbital }}$ and $\sigma_{\text {spin }}$ are directly related to the chemical shift and Knight Shift contributions respectively. They have the same scaling properties with the magnetic field.

\section{Results and discussion}

\subsection{X-ray diffraction}

Two different forms of crystalline GeTe can exist at room temperature, depending on the composition of the sample. The most well-known form is $\alpha-G e T e$, which is the stable form for the stoichiometric compound. It is supposed to be stable up to 50 at.\% Te [41] or 50.5 at.\% Te [42] (depending on the sources) and is a rhombohedral phase belonging to the R3m (160) space group. In this structure, the local symmetry around the Te atom is $3 \mathrm{~m}$, and it is surrounded by Ge atoms forming a slightly distorted octahedron. When there is 
an excess of Tellurium in the GeTe sample, starting from around 51 at.\% Te, the orthorhombic $\gamma$-GeTe is supposed to be the stable phase. This phase is less documented in the literature; it belongs to the Pnma (62) space group and is a binary analogous to the GeS type structure [41]. In this structure, the local structure around the Te atom is m, and the Te atom's first neighbors are also Ge atoms forming a distorted octahedron. Between 50 at.\% Te and 51 at.\% Te both phases can coexist.

Figure 2 shows X-ray diffractograms of the nominal $\mathrm{Ge}_{50} \mathrm{Te}_{50}$ and $\mathrm{Ge}_{48} \mathrm{Te}_{52}$ crystalline samples. $\mathrm{Ge}_{50} \mathrm{Te}_{50}$ appears to be single phase and the diffraction peaks correspond to $\alpha$-GeTe phase. With the increase of the tellurium content $\left(\mathrm{Ge}_{48} \mathrm{Te}_{52}\right)$ the instability of $\alpha$-GeTe increases, the main peak is slightly shifted and new diffraction peaks appeared. These new peaks correspond to the orthorhombic $\gamma$-GeTe phase and the hexagonal Te phase (space group P312). Thus, the $\mathrm{Ge}_{48} \mathrm{Te}_{52}$ crystalline sample is likely a mix of $\alpha-\mathrm{GeTe}$, $\gamma$-GeTe and a small amount of elemental Te crystalline phases. The presence of $\alpha$-GeTe, which is not the stable phase at this composition, is probably due to the fact that the sample has not reached equilibrium.

\subsection{Ab initio calculation in VCA approximation}

Many ab initio codes allow to calculate the shielding tensor $\sigma$ in crystalline system using periodic limit conditions and DFT. The shielding tensor $\sigma$ is related to the NMR shift $\delta$ thanks to the relation:

$$
\delta=\sigma_{\text {ref }}-\sigma
$$

where $\sigma_{r e f}$ is the isotropic shielding of the reference sample. In our case we are only interested in the behavior of the NMR shift when the amount of vacancies is varied in the $\mathrm{Ge}_{48} \mathrm{Te}_{52}$ sample compared to GeTe without vacancies, and $\sigma_{r e f}$ was disregarded. On the spectra, if the shielding $\sigma$ decreases (increases) then the corresponding chemical shift will vary in the opposite direction and the NMR line position will shift to higher (lower) ppm values.

When defects such as vacancies are present, accurate calculations are computationally more challenging than in the perfect crystal. An alternative is the Virtual Crystal Approximation, a simple approach much 
less computationally demanding than supercells, but also less accurate. This approach does not take into account the distortion of the lattice due to the suppression of an atom and only considers one average site for the entire structure, so it cannot deliver exact values and distributions of the NMR shifts, but it can provide a broad trend. In a more rigorous study the use of supercells would be required to simulate those defects but this is not in the scope of this paper.

We have performed VCA calculations on $\alpha$-GeTe and $\gamma$-GeTe crystalline phases, because our XRD measurements show that both phases are present in $\mathrm{Ge}_{48} \mathrm{Te}_{52}$. It is well known that $\alpha-\mathrm{GeTe}$, even with a stoichiometric composition, is never free of Ge vacancies [32], and that each vacancy creates two holes in the valence band [35]. A value of around $p=110^{20} \mathrm{~cm}^{-3}$ is predicted [35] as close as possible to stoichiometry and by varying the composition of the sample, the measured charge carrier concentration can vary between $\mathrm{p}=1.410^{20} \mathrm{~cm}^{-3}$ and $\mathrm{p}=1310^{20} \mathrm{~cm}^{-3}$ according to the literature $[21,32,33,34]$. For our calculations, we have chosen to vary the number of electrons in the cell from 30 electrons per GeTe unit to 29.8 electrons in order to vary the concentration $\mathrm{p}$ in charge carrier (holes) from 0 to $3.510^{21} \mathrm{~cm}^{-3}$. We have obtained the charge carrier concentration by dividing the number of electrons removed from the cell (NE parameter in wien $2 \mathrm{k})$ by the volume of the rhombohedral cell $\left(0.05388 \mathrm{~nm}^{3}, 1 \mathrm{GeTe}\right.$ unit) for $\alpha$-GeTe or by the volume of the orthorhombic cell $\left(0.2147 \mathrm{~nm}^{3}, 4\right.$ GeTe units $)$ for $\gamma$-GeTe. Thus we have calculated the NMR shieldings on the range of concentrations present in the literature and even further to better observe the evolution of these shieldings. The results of these calculations are presented in Figure 3.

It appears that the evolution of the Isotropic shielding $\sigma_{\text {iso }}$ versus the charge carrier concentration of holes $\mathrm{n}$ in the two phases is very different, both in terms of behavior and amplitude. In the case of $\alpha$-GeTe the evolution is rather complex and nonlinear whereas it is more monotonous in the case of $\gamma$-GeTe.

For $\gamma$-GeTe, the orbital part of the shielding decreases monotonically from 2300ppm to around $1000 \mathrm{ppm}$ on the range of concentrations described in the literature $\left(0.5-1.310^{21} \mathrm{~cm}^{-3}\right)$ while the spin part stays in the range of a few tens of ppm over all the studied range. The behavior of the spin part is typical of what is usually observed in insulators and semi-conductors, and for those materials this contribution is often neglected in calculations. The evolution of the total shielding is quasi-exclusively due to the orbital part 
and has the same behavior. For this phase, the NMR line position shifts exclusively to higher ppm values when we vary the concentration of charge carriers.

For $\alpha-G e T e$, the orbital part of the shielding first increases from $2500 \mathrm{ppm}$ to around $3700 \mathrm{ppm}$ for a concentration close to $0.7510^{21} \mathrm{~cm}^{-3}$ and then decreases slowly until $3500 \mathrm{ppm}$ for $1.310^{21} \mathrm{~cm}^{-3}$, while the spin part monotonically decreases from 0 to around $-1300 \mathrm{ppm}$. Such a huge variation of 1300ppm for the spin part is unusual for insulators and semiconductors but can be observed in conductive materials. Between $0 \mathrm{~cm}^{-3}$ and $0.410^{21} \mathrm{~cm}^{-3}$ the total shielding is greater than in the perfect structure (Zone I on Figure 3.b) and the line position is shifted to lower ppm values up to $500 \mathrm{ppm}$ compared to the perfect structure. Between $0.410^{21} \mathrm{~cm}^{-3}$ and $0.910^{21} \mathrm{~cm}^{-3}$ the total shielding stays close to the value of the perfect structure (Zone II on Figure 3.b). For higher concentrations the total shielding is smaller than in the perfect structure (Zone III on Figure 3.b) and the line can be shifted to higher ppm values by several thousands of ppm until around $500 \mathrm{ppm}$ (for around $3.510^{21} \mathrm{~cm}^{-3}$ ).

According to Raty et al. [5], based on DFT calculations, the orthorhombic $\gamma$-GeTe phase possesses a covalent bonding while the $\alpha$-GeTe has a "metavalent bonding". The unusual behavior of both orbital and spin part in $\alpha$-GeTe could be a signature of the metavalent bonding while the more conventional one in $\gamma$ GeTe is consistent with a covalent bonding.

Our calculations allow to predict a full NMR shift range of at least $2800 \mathrm{ppm}$ on the range of charge carrier concentrations described in the literature and the NMR line position could shift to higher or lower ppm values on the spectra compared to the perfect structure, depending on the phase and the concentration of holes.

\section{3. ${ }^{125}$ Te NMR measurements}

\subsubsection{VOCS technique}

The VOCS technique has been applied to the $\mathrm{Ge}_{48} \mathrm{Te}_{52}$ sample with a dilution of 40 vol. $\% \mathrm{Ge}_{48} \mathrm{Te}_{52}-60$ vol. $\%$ $\mathrm{SiO}_{2}$ (see section 3.3) on the 14.1T magnet in order to compare with results of the literature [21]. 11 spectra were recorded for a total experiment duration of $11 \mathrm{~h}$ which does not include tuning and matching of the 
probe between each of them. In order to favorize $\mathrm{S} / \mathrm{N}$ ratio the full shifted echo where registered which correspond to an improvement of $\sqrt{2}$ compared to the registration of the FID synchronized on the top of the echo. Phasing of the spectra is also straightforward as the magnitude spectra can be used safely without loss of resolution [16]. This is a gain in robustness since conventional phasing can be tricky for wide spectra. The corresponding NMR spectra of $\mathrm{Ge}_{48} \mathrm{Te}_{52}$ are shown in Figure 4 together with the summation spectrum. The full spectrum is broad with an expansion over several thousands of ppm and is comprised in a spectral width window of less than $1.25 \mathrm{MHz}$. A maximum is observed close to $1500 \mathrm{ppm}$ and a tail is expanded to higher ppm values up to $5000 \mathrm{ppm}$. This result is consistent with the spectrum obtained by Levin et al. for a close composition [21]. It appears that this method produces spectra with rather poor signal-to-noise ratio.

\subsubsection{Low field experiments (2.35T and 9.4T)}

We have acquired a NMR spectrum of stoichiometric GeTe sample at low frequency using CPMG technique to determine if the use of lower frequency would be better suited to our study. The purpose of using the low 2.35T field was that at such a magnetic field the width of the spectrum connected to isotropic chemical shift distribution and anisotropy is 6 times lower than at 14.1T. It enables to use Magic Angle Spinning (30kHz) in this sample because the linewidth is reduced, and also WURST pulses are no longer required.

Figure 5 presents the comparison between the spectra of the $\mathrm{Ge}_{50} \mathrm{Te}_{50}$ crystalline sample acquired at $2.35 \mathrm{~T}$ with MAS and 14.1T in static conditions. While MAS suppresses anisotropy leading to an improvement of the intensity (no spinning sidebands are observed), it is obvious that the $12 \mathrm{~h} 14.1 \mathrm{~T}$ spectrum has a better $\mathrm{S} / \mathrm{N}$ than the $28 \mathrm{~h} 2.35 \mathrm{~T}$ one.

In Figure 6.a) we present the comparison between the spectra of the $\mathrm{Ge}_{48} \mathrm{Te}_{52}$ crystalline sample acquired at 9.4T and $14.1 \mathrm{~T}$ both in static conditions and with the WURST-CPMG technique. The same rotor was used for both measurements.

It is trickier to determine which of the $9.4 \mathrm{~T}$ or $14.1 \mathrm{~T}$ magnet gives the best results. On the normalized spectra, when we zoom in on the noise at the edge of the spectra, we can observe that the level of noise is slightly higher for the $9.4 \mathrm{~T}$ spectrum. The signal-to-noise ratio is equal to 160 for the $14.1 \mathrm{~T}$ and 120 for the 9.4T. This is a general observation we made on various samples. Moreover, we can better observe the low 
intensity tail of the signal at high ppm values in $\mathrm{Ge}_{48} \mathrm{Te}_{52}$ on the $14.1 \mathrm{~T}$ spectrum. This may be related to the characteristics of the RF circuitry of the two probes operating respectively at $189.24 \mathrm{MHz}$ and $126.16 \mathrm{MHz}$. Thus, among the 3 magnets that were tested the field of $14.1 \mathrm{~T}$ is the best suited to our experiments and we will focus solely on experiments carried out at $14.1 \mathrm{~T}$ for the rest of the discussion.

\subsubsection{WURST-CPMG technique}

We have compared the VOCS method with WURST-CPMG, which is a less constraining method to record wide spectra. Our goal was to optimize this technique in order to obtain the best Signal-to-Noise ratio $(\mathrm{S} / \mathrm{N})$ with reasonable experiment duration, at least less than one night or $12 \mathrm{~h}$.

The samples are conductive (around $510^{4} \mathrm{~S} / \mathrm{cm}$ ) which causes instability of the probe: tuning and matching are impossible if rotors are filled with the telluride sample powder alone. That is why we diluted our $\mathrm{Ge}_{48} \mathrm{Te}_{52}$ samples with $\mathrm{SiO}_{2}$ powder. We carried out a systematic study of the dilution of the sample from $20 \%$ in volume to $80 \%$ in order to determine which concentration would yield the best $\mathrm{S} / \mathrm{N}$. If the concentration in $\mathrm{Ge}_{48} \mathrm{Te}_{52}$ is too low, the signal isn't intense enough and $\mathrm{S} / \mathrm{N}$ is poor. However, if the concentration is too important, the tuning and matching of the probe is tricky, which impacts the quality and accuracy of the final spectrum. For each dilution, we have also optimized the power of the RF pulses to maximize signal intensity [37].

This study has been performed for different diameters of the rotors by using different probes of similar technology. We used thin wall $(36 \mu \mathrm{L})$ and standard $(22 \mu \mathrm{L}) 3.2 \mathrm{~mm}$ rotors, $2.5 \mathrm{~mm}(11 \mu \mathrm{L})$ and $1.6 \mathrm{~mm}(8 \mu \mathrm{L})$ rotors. For each configuration we first acquired a 3 h30 spectrum. The spectra resulting from these optimizations are presented in Figure 7 and the corresponding $\mathrm{S} / \mathrm{N}$ of all the spectra are reported in Figure 8.

When the inner diameter of the rotor is reduced, the volume of powder introduced in the rotor is reduced in the same proportion. This should lead to lower $\mathrm{S} / \mathrm{N}$ for smaller diameter. However, with such small rotors, in particular for $1.6 \mathrm{~mm}$ probe, we were able to use higher $\mathrm{Ge}_{48} \mathrm{Te}_{52}$ concentrations (up to $80 \%$ ) without disrupting the probe which can compensate partially. Moreover, RF circuitry can be optimized differently by the constructor and a higher quality factor of the probe for smaller diameter may also 
compensate the loss of sensitivity due to smaller amount of sample. For instance, the $3.2 \mathrm{~mm}$ and 1.6 probes have similar sensitivity on the constructor data sheet despite the difference of sample volume.

A surprising phenomenon is that we observed a maximum of the signal and signal to noise ratio in the $1.6 \mathrm{~mm}$ probe for $60 \mathrm{vol} \% \mathrm{Ge}_{48} \mathrm{Te}_{52}$ which contains less sample than the $80 \mathrm{vol} \% \mathrm{Ge}_{48} \mathrm{Te}_{52}$. It means the efficiency of the system deteriorates when the concentration approaches $100 \mathrm{vol} \%$. This phenomenon starts to appear also for the 2.5 probe, where the maximum intensity doesn't evolve from $50 \mathrm{vol} \%$ to $60 \mathrm{vol} \%$. One possible explanation can come from the skin effect or depth penetration distance of an electromagnetic wave in a conductive material. If the dilution is low the mean conductivity of the powder can increase and it is possible that a part of the sample is not reached.

When comparing Figure 7 (a) and Figure 7 (b), we obtained the best $\mathrm{S} / \mathrm{N}$ with the $3.2 \mathrm{~mm}$ probe using a thin wall rotor. The difference in intensity between a thin wall rotor and a standard rotor is roughly proportional to the difference in sample quantity inside the rotor which is largely favorable to the thin wall rotor, hence only the thin wall results are considered on $3.2 \mathrm{~mm}$ probe.

Another issue can be observed in Figure 9 where the best spectra for the 3 probes are plotted after normalization of the maximum intensity. It appears that the shapes of the envelope of the full spectra are slightly different. The main effect is that the tail of the signal at high chemical shift values is much better observed in the $3.2 \mathrm{~mm}$ thin wall probe than in the others. We relate this to the higher quality factors of the smaller probes designed to compensate loss of sensitivity but that consequently modify unfavorably the width of the bandwidth. The excitation profile of the 1.6 and $2.5 \mathrm{~mm}$ probes is then less favorable to wide spectra.

The conclusion of this part is that the best results are obtained with the $3.2 \mathrm{~mm}$ probe equipped with thin wall rotors. In the following of the study the $40 \%$ sample dilution will be retained in order to secure tuning and matching operations.

\subsection{4. pj-MAT experiments}


The 2D pj-MAT maps for $\mathrm{Ge}_{50} \mathrm{Te}_{50}$ and $\mathrm{Ge}_{48} \mathrm{Te}_{52}$ are presented in Figure 10.a). These maps allow us to separate the Chemical Shift Anisotropy (CSA) in the dimension F2 from the isotropic chemical shift in the second F1 dimension.

For $\mathrm{Ge}_{50} \mathrm{Te}_{50}$, two distinct distributions can be observed: a distribution of isotropic chemicals shifts visible along the chemical shift axis which corresponds to the diagonal of the figure, which is visible on the projection on the Isotropic Chemical Shift axis (Figure 11), accompanied by a distribution of anisotropy observed on the F1 slices. To quantify this anisotropy, we have extracted seven horizontal slices from the $\mathrm{Ge}_{50} \mathrm{Te}_{50}$ 2D spectrum, which we have fitted with the Dmfit [43] software and presented in Figure 12. All these slices can be fitted easily with a static Chemical Shift Anisotropy model with axial symmetry. This is coherent with the $3 \mathrm{~m}$ local symmetry around the Te atom in the perfect $\alpha$-GeTe structure without vacancies. The experimental slices are slightly distorted compared to the theoretical model due to the behavior of the magnetization during the pulse sequence. $\delta_{/ /}$and $\delta_{\perp}$ can then be represented on the 2D map. This allows us to plot two axes associated to the evolution of these values on each side of the chemical shift axis. This highlights the correlation between anisotropy and isotropic line position. An increase of the isotropic value of the chemical shift coincides with a decrease of the anisotropy. The anisotropy can even vanish for high values of the Isotropic Chemical Shift. This decrease of the anisotropy could be tentatively related to some motional narrowing induced by the presence of Ge vacancies that allow Ge atoms to jump from occupied to unoccupied sites. The increase of the concentration of Ge vacancies would lower the mean anisotropy and increase the isotropic chemical shift.

For $\mathrm{Ge}_{48} \mathrm{Te}_{52}$, only a distribution of isotropic chemical shifts can be observed along the chemical shift axis, also visible on the projection on the Isotropic Chemical Shift axis (Figure 11). We also have extracted six horizontal slices for this system: only one peak is visible on each spectrum, whose position corresponds to the isotropic chemical shift value of the slice. This indicates that no anisotropy is present. An attempt to introduce anisotropy in the fit in order to account for the linewidth was made. This led to isotropic chemical shift modifications which were not compatible with the 2D map, hence the spectra are well fitted with a simple Lorentzian curve, which is usually invoked when dynamic processes are involved. Thus, only an isotropic chemical shift distribution is present in this sample. This is consistent with the results obtained for 
$\mathrm{Ge}_{50} \mathrm{Te}_{50}$ but with a larger amount of vacancies, associated with a Te-rich composition, which would allow for stronger motional narrowing and a disappearance of the anisotropy.

In Figure 13 the Anisotropic Chemical Shift projections of the pj-MAT spectra of both samples are superimposed to the WURST-CPMG spectra. In WURST-CPMG, the pulses used are adiabatic and they make it possible to irradiate a broader range of frequencies than in standard CPMG. For $\mathrm{Ge}_{50} \mathrm{Te}_{50}$, the spectra recorded with the two techniques are in adequation. For $\mathrm{Ge}_{48} \mathrm{Te}_{52}$, the duration of the CPMG pjMAT pulses doesn't allow us to recover the totality of the WURST-CPMG spectrum. Hence, two experiments with two different offsets were performed (blue and purple). A third experiment with a higher offset was also performed but the signal was lost in the noise (not represented). Taking into account the poor signal-to-noise ratio, the pj-MAT projections and the WURST-CPMG spectrum are also in adequation.

\subsubsection{Comparison between Ge50 $\mathrm{Te}_{50}$ and $\mathrm{Ge}_{48} \mathrm{Te}_{52}$ crystalline samples}

Figure 13 presents a comparison of the spectra of $\mathrm{Ge}_{50} \mathrm{Te}_{50}$ and $\mathrm{Ge}_{48} \mathrm{Te}_{52}$ crystalline samples acquired with WURST-CPMG with a dilution of $40 \%$ with $3.2 \mathrm{~mm}$ thin-wall rotors over 12 hours for best signal-to-noise ratios.

We can observe that the signal for $\mathrm{Ge}_{50} \mathrm{Te}_{50}$ is slightly dissymmetric, with a shoulder on the right of the maximum. For this composition, XRD measurements show that only $\alpha-G e T e$ is present in the material. If we consider that the maximum of the distribution corresponds to the signal of perfect $\alpha$-GeTe without vacancies, this shoulder on the right could correspond to $\alpha$-GeTe with some vacancies. This is consistent with VCA calculations which predict that for a relatively low amount of vacancies (inferior to $0.210^{21} \mathrm{~cm}^{-}$ ${ }^{3}$ ), the NMR position of $\alpha$-GeTe should shift to lower ppm values, and with the fact that stoichiometric GeTe never exists without vacancies.

For $\mathrm{Ge}_{48} \mathrm{Te}_{52}$, there is a global shift of the maximum of the signal of more than 500 ppm compared to $\mathrm{Ge}_{50} \mathrm{Te}_{50}$. This shift can also be observed on the Isotropic Chemical Shift projection of the pj-MAT spectra. This could correspond to the transition from $\alpha$-GeTe to $\gamma$-GeTe, since $\gamma$-GeTe is supposed to be the stable phase at this composition, and the signal of perfect $\gamma$-GeTe is shifted to higher ppm values compared to 
perfect $\alpha$-GeTe according to VCA calculations (see Figure 3.b). However, XRD measurements suggest that $\mathrm{Ge}_{48} \mathrm{Te}_{52}$ is a mix of $\alpha$-GeTe, $\gamma$-GeTe and Te. The shift to higher ppm values could then also correspond to the presence of $\alpha$-GeTe containing a large number of vacancies (superior to $0.4510^{21} \mathrm{~cm}^{-3}$ ). The results of the pj-MAT investigations can complement these interpretations. We have concluded that a shift to higher ppm values of the NMR line position was mainly associated with an increase of the concentration in vacancies in both $\alpha$ and $\gamma$ structures. $\delta_{/ /}$and $\delta_{\perp}$ axis in the pj-MAT maps suggest that the isotropic chemical shift range associated with high concentrations of Ge vacancies in $\alpha-G e T e$ lead to the vanishing of the anisotropy. Hence $\alpha$ and $\gamma$ contributions will be superimposed in $\mathrm{Ge}_{48} \mathrm{Te}_{52}$. The $\mathrm{Ge}_{48} \mathrm{Te}_{52}$ sample is thus rather complex, which explains the width of the Isotropic Chemical Shift projection of its pj-MAT spectrum, compared to the $\mathrm{Ge}_{50} \mathrm{Te}_{50}$ projection which is narrower and well defined.

In the WURST-CPMG spectra, we can also notice that the spectrum of $\mathrm{Ge}_{48} \mathrm{Te}_{52}$ is significantly larger than the one of $\mathrm{Ge}_{50} \mathrm{Te}_{50}$. The pj-MAT spectrum shows that this is only due to a distribution of $\delta_{\text {iso }}$ and not to the anisotropy. The WURST-CPMG spectrum possesses a wide "tail" that extends up to 4500 ppm higher than the maximum of the signal. It cannot be observed in pj-MAT measurements because of its small intensity and the poor signal-to-noise ratio of the pj-MAT. This "tail" is probably due to a distribution of concentrations of charge carriers in the sample, because it likely contains different zones with varying concentrations of vacancies. According to VCA calculations, this tail could correspond to $\alpha$-GeTe with a high amount of vacancies and $\gamma$-GeTe with any amount of vacancies. It is important to note that in our calculations, we do not have such a wide extension of at least 4500ppm at higher ppm values. This could be due to the inaccuracy of the VCA method, and it will be interesting to use more accurate calculation methods to pursue this study.

The shape of the spectrum of $\mathrm{Ge}_{48} \mathrm{Te}_{52}$ is in accordance with the results of Levin et al [14]. In their article they confirmed experimentally that it is possible to estimate the charge carrier density thanks to $T_{1}$ measurements and the variations of $\mathrm{T}_{1}$ lead them to also attribute the extension of the spectrum to higher ppm values to a distribution of concentrations of charge carriers in the material. 
However, a difference with our analysis is that in Levin's article the extension of the spectrum is related to a variation of the Knight Shift (the spin part of the NMR shift). Yet our calculations seem to suggest that the orbital part is hugely responsible for the variations of the NMR Shift, in both $\alpha$-GeTe and $\gamma$-GeTe.

\section{Conclusion}

Crystalline $\mathrm{Ge}_{50} \mathrm{Te}_{50}$ and $\mathrm{Ge}_{48} \mathrm{Te}_{52}$ were synthesized in sealed silica ampoules. XRD measurement showed that $\mathrm{Ge}_{50} \mathrm{Te}_{50}$ was single phase $(\alpha-\mathrm{GeTe})$ and $\mathrm{Ge}_{48} \mathrm{Te}_{52}$ was a mix of $\alpha-\mathrm{GeTe}, \gamma-\mathrm{GeTe}$ and a small amount of elemental Te crystalline phases.

${ }^{125}$ Te $1 \mathrm{D}$ NMR experiments have shown that the WURST-CPMG technique is an efficient method to record wide spectra in PCM where vacancies are present and the material is conductive. It allowed to record wide spectra with better $\mathrm{S} / \mathrm{N}$ ratios than the VOCS technique usually used and with much shorter experiment durations within a couple of hours. In addition, the WURST-CPMG technique showed to be more robust since only one tuning and matching is required for each spectrum. We concluded that a $40 \%$ dilution by volume is a good compromise between signal-to-noise ratio and easy tuning and matching. The most suitable probe was the standard $3.2 \mathrm{~mm}$ probe used with thin wall rotors. It was preferable to use a $14.1 \mathrm{~T}$ magnetic field rather than a lower magnetic field $(2.35 \mathrm{~T}$ or $9.4 \mathrm{~T})$. The combination of a $14.1 \mathrm{~T}$ magnetic field with $3.2 \mathrm{~mm}$ probe and thin wall rotor was even better than an experiment on a $2.35 \mathrm{~T}$ which allows using MAS and standard pulses but presents an important loss of sensitivity. It is particularly true in systems where the linewidth is mainly due to chemical shift distributions due to defects such as vacancies for which MAS standard experiments (single pulse, echoes...) are not efficient.

For $\mathrm{Ge}_{50} \mathrm{Te}_{50}$, the WURST-CPMG spectrum is relatively simple, when for $\mathrm{Ge}_{48} \mathrm{Te}_{52}$ it is more complex and asymmetric, with a long "tail" observed at high values of the chemical shift scale.

${ }^{125}$ Te NMR 1D experiments were combined with predictions of NMR shift using the VCA model in WIEN2k which allowed to link the lineshape of the spectra to a distribution of charge carriers in the material. A shift of the NMR line position to higher chemical shift values is connected to higher amounts of vacancies. Although some trends were observed, the VCA method is approximative and it would be interesting to 
perform more accurate $\mathrm{ab}$ initio calculations using various supercell sizes to obtain more realistic values of the NMR shifts taking into account structural relaxation and depicting different vacancy concentrations. 2D pj-MAT experiments using 10kHz MAS, allowed us to distinguish between the isotropic and anisotropic chemical shift distributions. In $\mathrm{Ge}_{50} \mathrm{Te}_{50}$ the two types of distribution were evidenced. In this phase the amount of vacancies is low and it is close to the "perfect" $\alpha-$ GeTe structure. However, the sample seems to be inhomogeneous and the anisotropy decreases when the concentration in vacancies increases. This can be related to the presence of a vacancy in the vicinity of the Te atom, allowing the first neighbors Ge atoms to jump from an occupied site to a vacancy, thus creating an average close to cubic symmetry around Te. In $\mathrm{Ge}_{48} \mathrm{Te}_{52}$, only an isotropic chemical shift distribution is observed, together with a global shift of the line position. This is consistent with our interpretation for $\mathrm{Ge}_{50} \mathrm{Te}_{50}$, but for a higher Ge-deficient composition. By combining the WURST-CPMG technique with the pj-MAT technique, we have been able to detect efficiently and in a reasonable amount of time small signals associated to vacancies, but also NMR parameters distribution.

\section{Acknowledgments}

The authors would like to thank P. Sans (ICGM Montpellier, France) for his help in setting up the WIEN2K software and Z. Gan (National High Magnetic Field Laboratory, Tallahassee, USA) for answering questions about the pj-MAT sequence implementation. 


\section{Figures}

Figure 1: Pulse sequences for a) WURST-CPMG technique [37] and b) pjMAT technique [29]

Figure 2: X-ray diffractograms of the $\mathrm{Ge}_{50} \mathrm{Te}_{50}$ and $\mathrm{Ge}_{48} \mathrm{Te}_{52}$ crystalline phases. Reflexions corresponding to $\alpha-\mathrm{GeTe}, \gamma$-GeTe and Te crystalline phases are indicated.

Figure 3: VCA calculation of isotropic shielding vs defect concentration a) orbital (circle) and spin (triangle) contributions and $b$ ) total contribution for $\alpha$-GeTe (orange) and $\gamma$-GeTe (purple). The crosshatched zone corresponds to the concentrations described in the literature. The horizontal orange and purple line correspond to the values for the perfect structure for each curve. Compared to the stoichiometric GeTe in $\alpha$-GeTe the NMR line is shifted on the left and on the right in regions I and III respectively, almost unshifted in region II, always on the left for $\gamma$-GeTe

Figure 4: ${ }^{125} \mathrm{Te} \mathrm{NMR}$ spectrum of $\mathrm{Ge}_{48} \mathrm{Te}_{52}$ made with the VOCS method (in color : individual contributions, in black: sum of the individual contributions)

Figure 5: a) ${ }^{125} \mathrm{Te}$ NMR spectra of $\mathrm{Ge}_{50} \mathrm{Te}_{50}$ for a) $2.35 \mathrm{~T}$ (100MHz, top) magnet using MAS and CPMG techniques and b) for 14.1T (600MHz, bottom) magnet using static WURST-CPMG technique. b) WURSTCPMG pulse sequence diagram.

Figure 6: ${ }^{125} \mathrm{Te}$ WURST-CPMG of $\mathrm{Ge}_{48} \mathrm{Te}_{52}$ for $9.4 \mathrm{~T}$ (400MHz, top) and $14.1 \mathrm{~T}$ (600MHz, bottom) magnetic fields. Insert: noise in high field region.

Figure 7: ${ }^{125} \mathrm{Te}$ NMR spectra of $\mathrm{Ge}_{48} \mathrm{Te}_{52}$ for every dilution in volume: (-) $20 \%$ - (一) $30 \%$ - (一) $40 \%$ - (-) $50 \%-(-) 60 \%-(-) 70 \%-(-) 80 \%$ a) Thin wall $3.2 \mathrm{~mm}$ probe rotor $-\mathrm{b})$ Standard $3.2 \mathrm{~mm}$ probe 
rotor $-\mathrm{c}$ ) Standard $2.5 \mathrm{~mm}$ probe - d) Standard $1.6 \mathrm{~mm}$ probe. Shift between each spectrum: 5 a.u. vertically, 2500 ppm horizontally

Figure 8: Signal to Noise $(\mathrm{S} / \mathrm{N})$ for every experiment - square $: 3.2 \mathrm{~mm}$ thin wall, triangle $: 2.5 \mathrm{~mm}$ std, circle : $3.2 \mathrm{~mm}$ std, diamond : $1.6 \mathrm{~mm}$ std.

Figure 9: ${ }^{125} \mathrm{Te}$ NMR spectra of $\mathrm{Ge}_{48} \mathrm{Te}_{52}$ with optimized parameters for each probe from left to right: $3.2 \mathrm{~mm}$ standard, $3.2 \mathrm{~mm}$ thin wall, $2.5 \mathrm{~mm}$ standard, $1.6 \mathrm{~mm}$ standard (shift between each spectrum: 0,1 a.u. vertically, 3500 ppm horizontally).

Figure 10: a) 2D pj-MAT spectra of $\mathrm{Ge}_{50} \mathrm{Te}_{50}$ (red) and $\mathrm{Ge}_{48} \mathrm{Te}_{52}$ (two offsets: blue at 1394 ppm and purple at $1973 \mathrm{ppm}$ ) crystalline samples. The solid black line represents the chemical shift axis, the solid dark red lines represent $\delta_{/ /}$and $\delta_{\perp}$. The dashed red and blue lines represent the position of the different F2 slices. b) pj-MAT pulse sequence diagram.

Figure 11: projection of the pj-MAT spectra of $\mathrm{Ge}_{50} \mathrm{Te}_{50}$ (red) and $\mathrm{Ge}_{48} \mathrm{Te}_{52}$ (blue at 1394 ppm) on the Isotropic Chemical Shift axis.

Figure 12: F2 Slices extracted from the 2D pj-MAT spectrum of $\mathrm{Ge}_{50} \mathrm{Te}_{50}$ (red) and $\mathrm{Ge}_{48} \mathrm{Te}_{52}$ (blue) with simulated spectra (black). Isotropic value, from top to bottom, Ge $\mathrm{F}_{50} \mathrm{Te}_{50}: 675 \mathrm{ppm}, 720 \mathrm{ppm}, 765 \mathrm{ppm}, 810$ ppm, 855 ppm, 900 ppm, 945 ppm; Ge $48 \mathrm{Te}_{52}$ : 1100 ppm, 1200 ppm, 1300 ppm, 1400 ppm, 1500 ppm, 1600 ppm.

Figure 13: ${ }^{125} \mathrm{Te}$ NMR WURST-CPMG spectra of $\mathrm{Ge}_{50} \mathrm{Te}_{50}$ (red) and $\mathrm{Ge}_{48} \mathrm{Te}_{52}$ (two offsets: blue at 1394 ppm and purple at $1973 \mathrm{ppm}$ ) superimposed with F1 projections on the anisotropic chemical shift axis from the pj-MAT, together with elemental Te (black). 
Figure 1

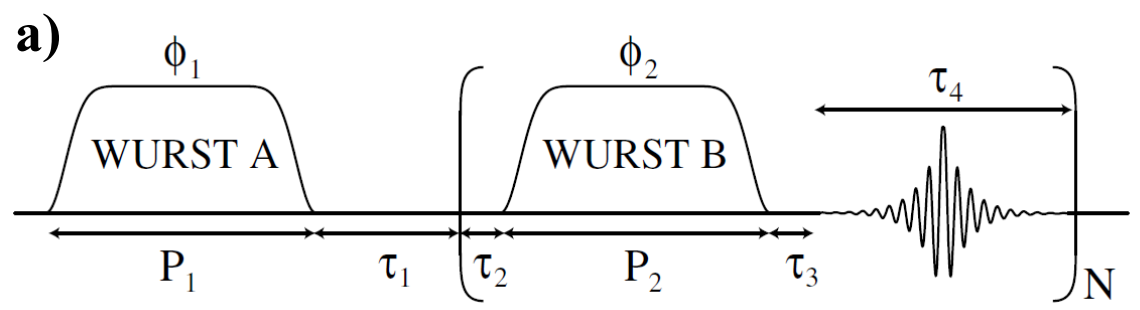

b)

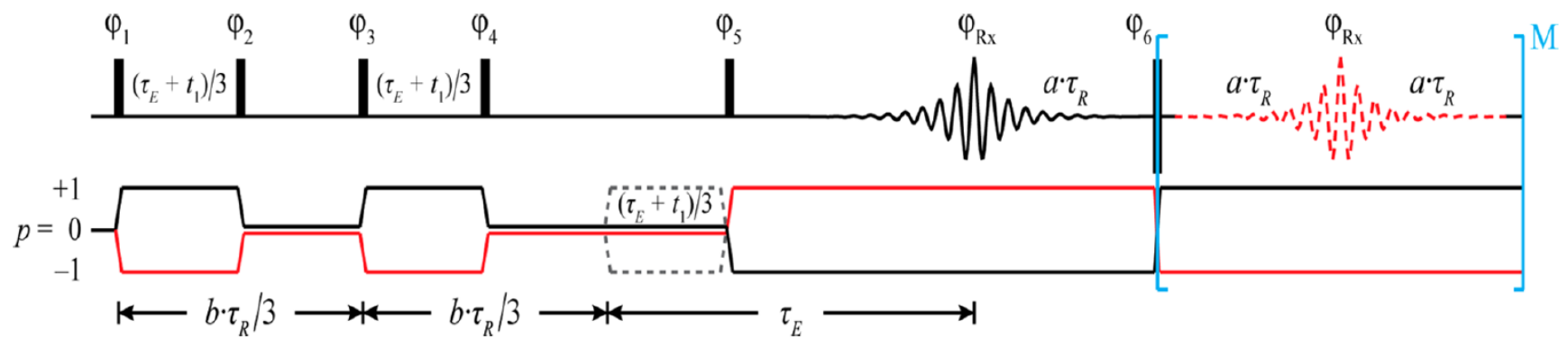


Figure 2

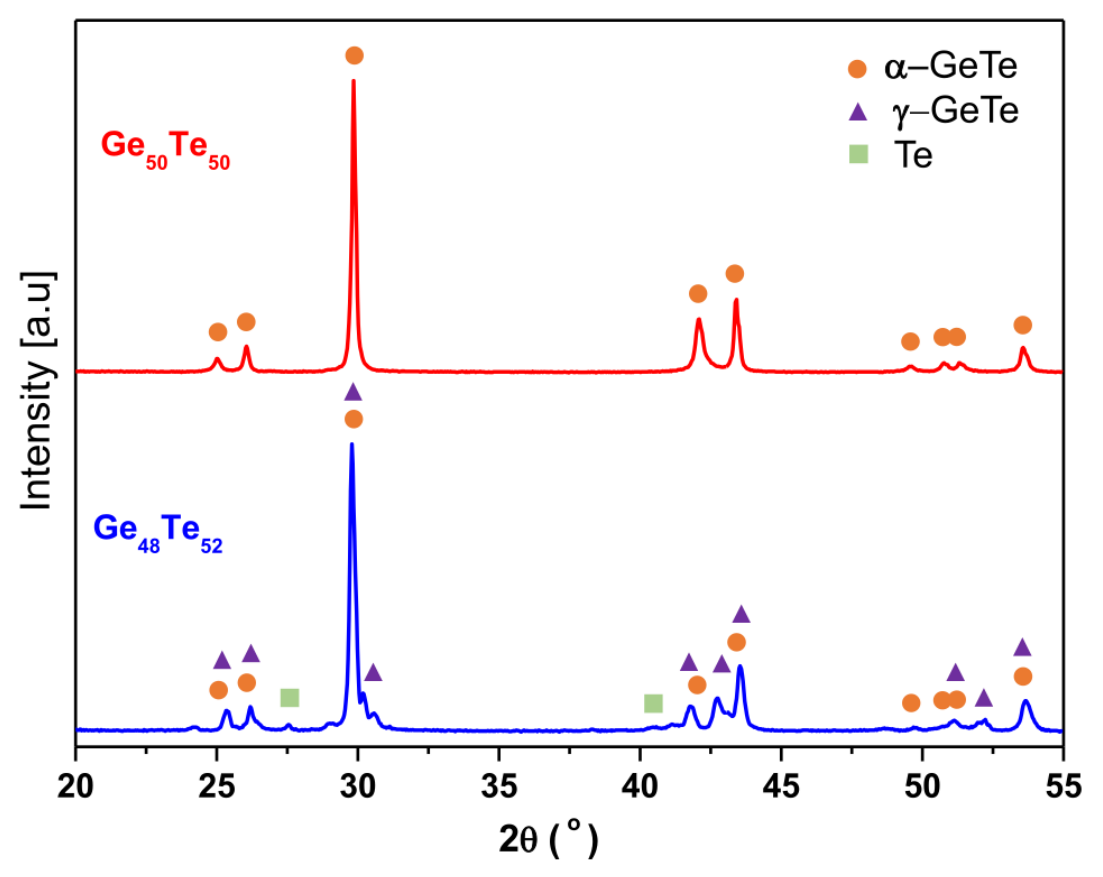


Figure 3
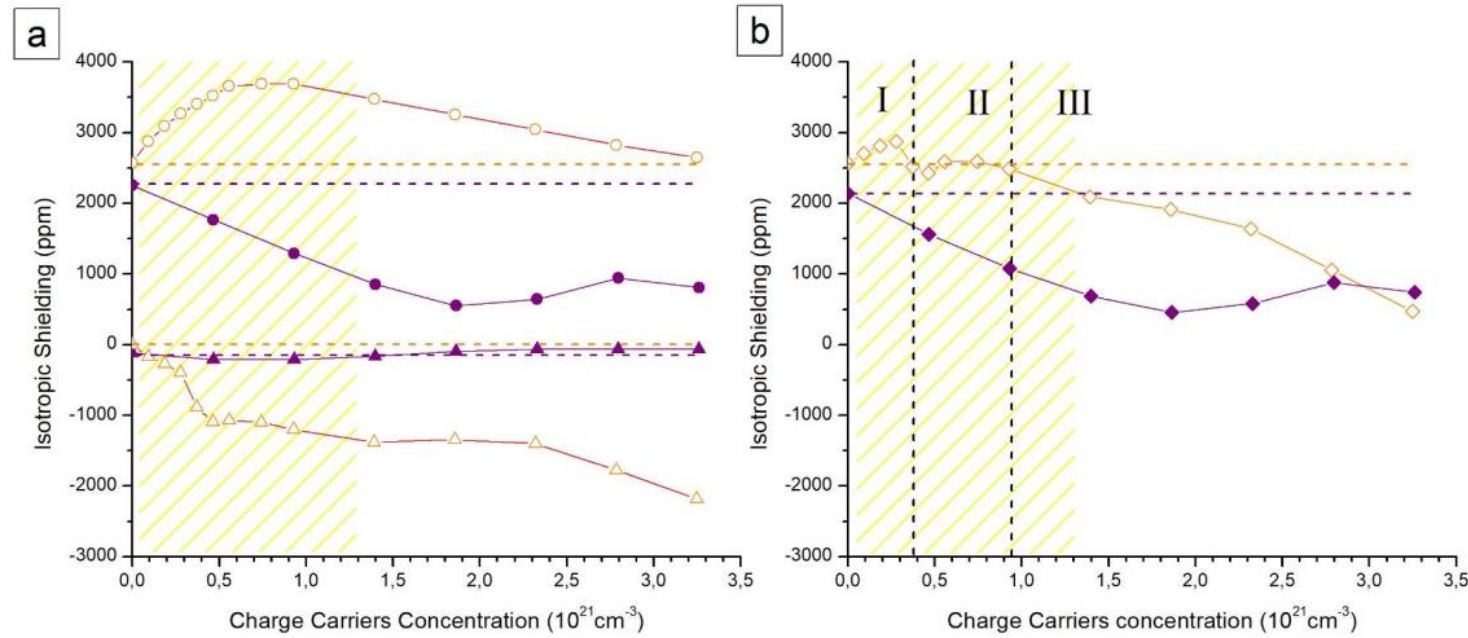
Figure 4

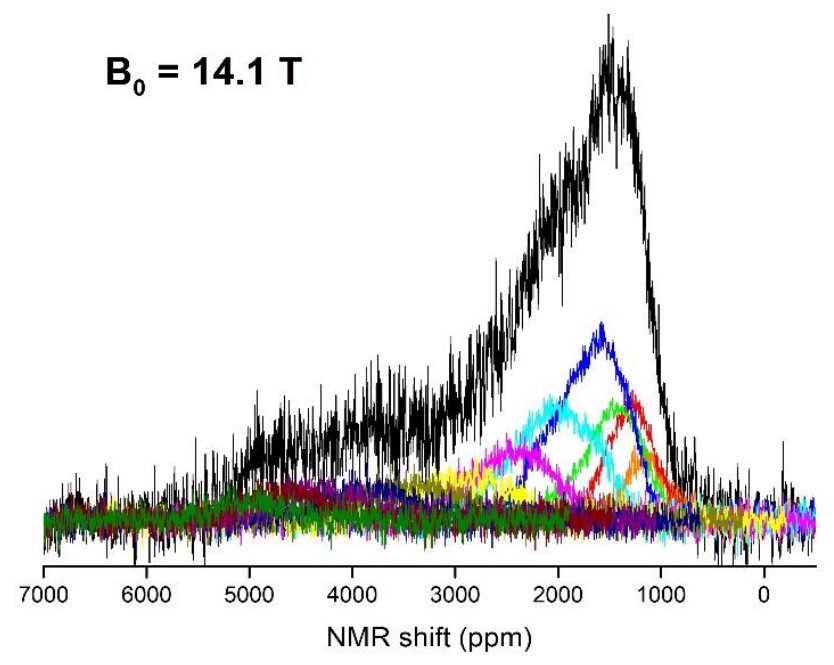


Figure 5

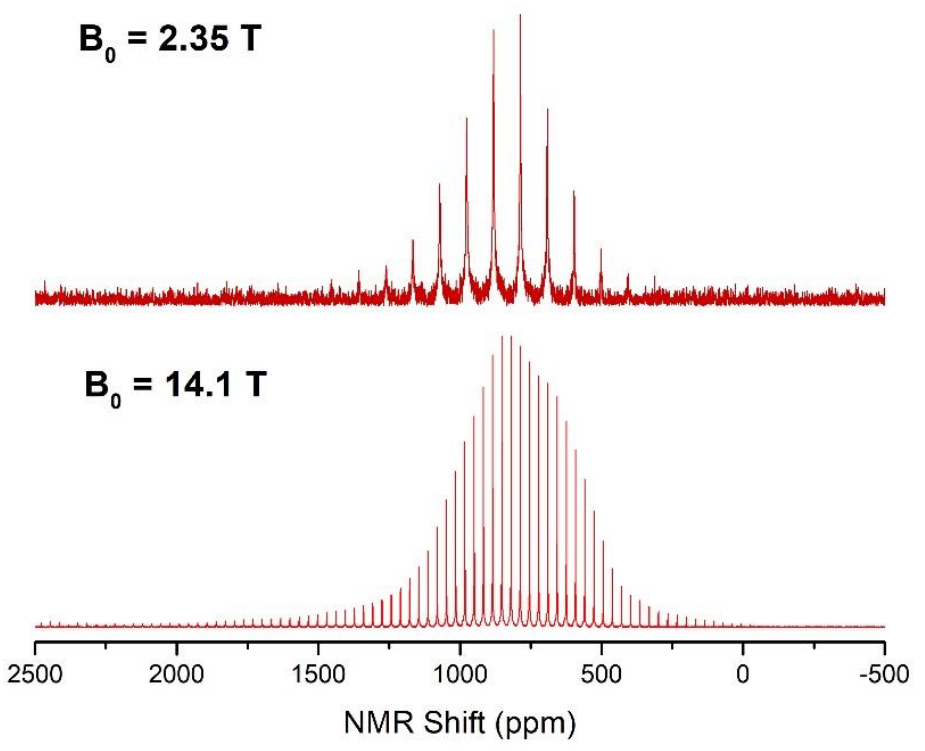


Figure 6

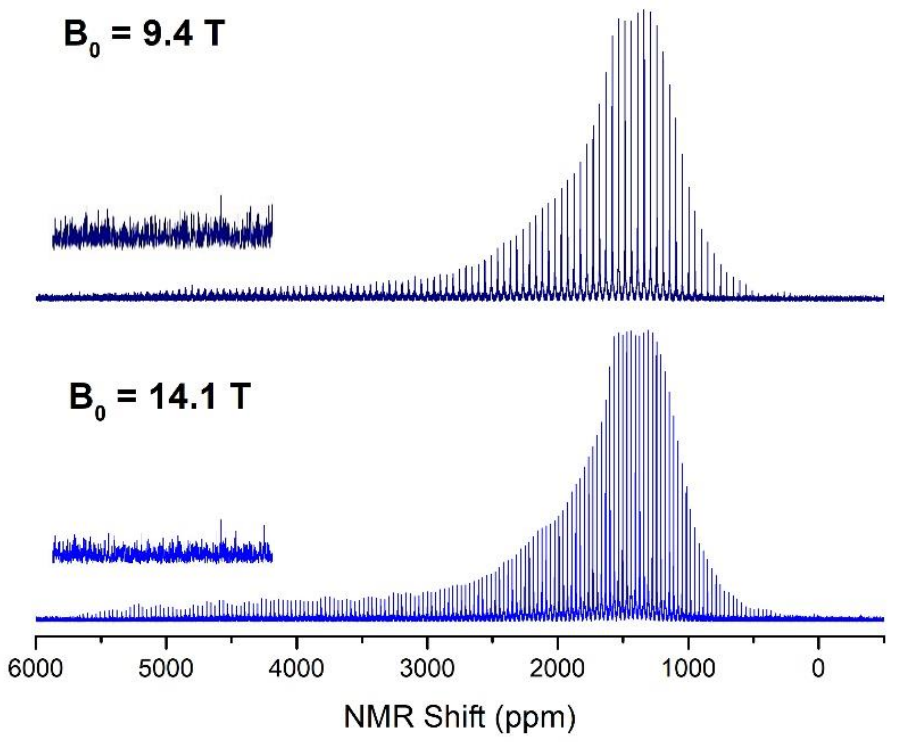


Figure 7
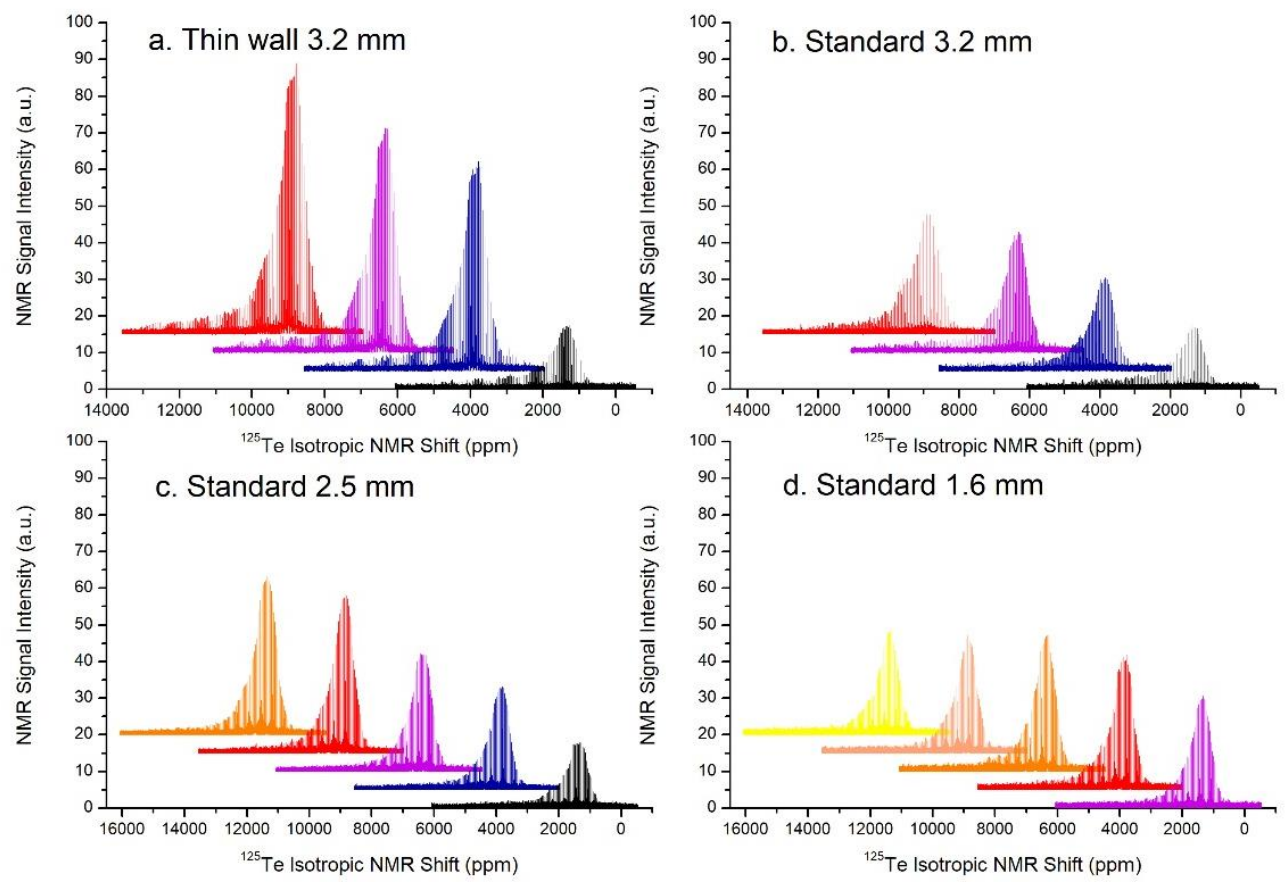
Figure 8

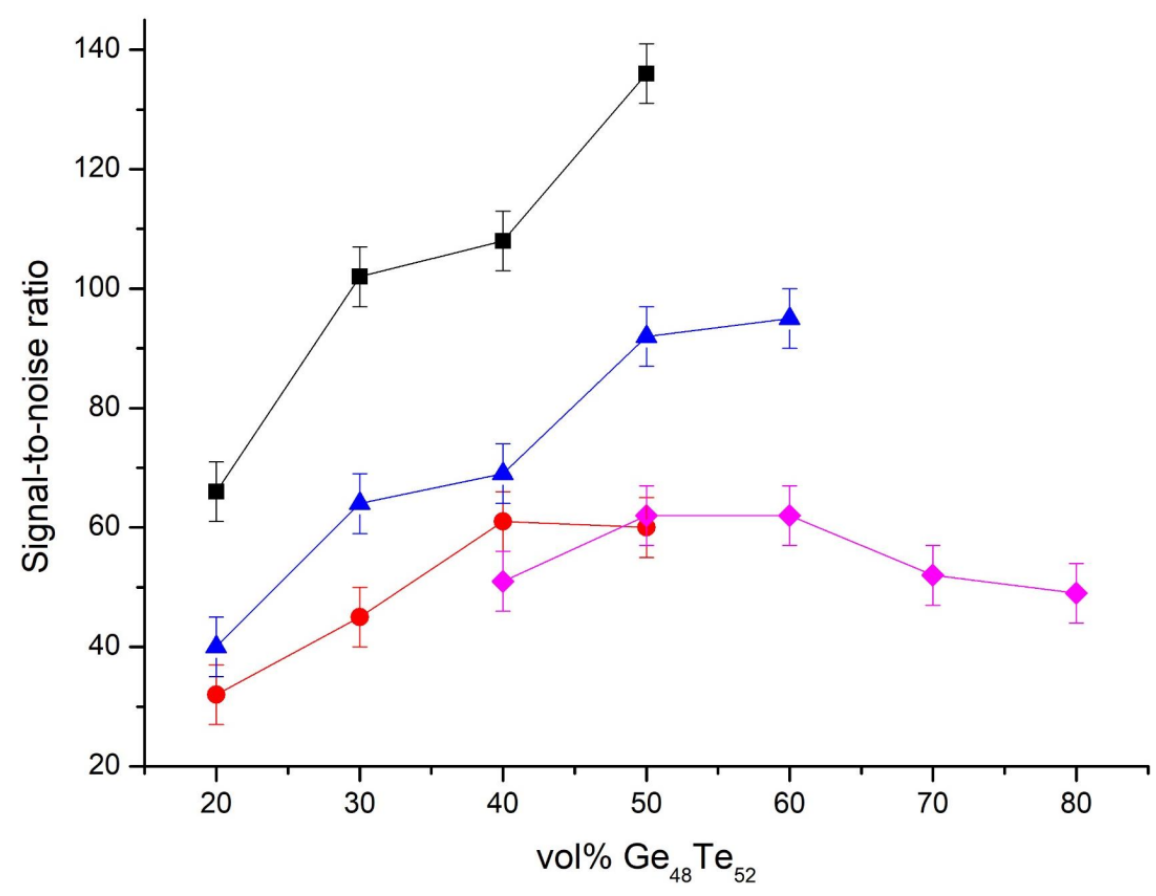


Figure 9

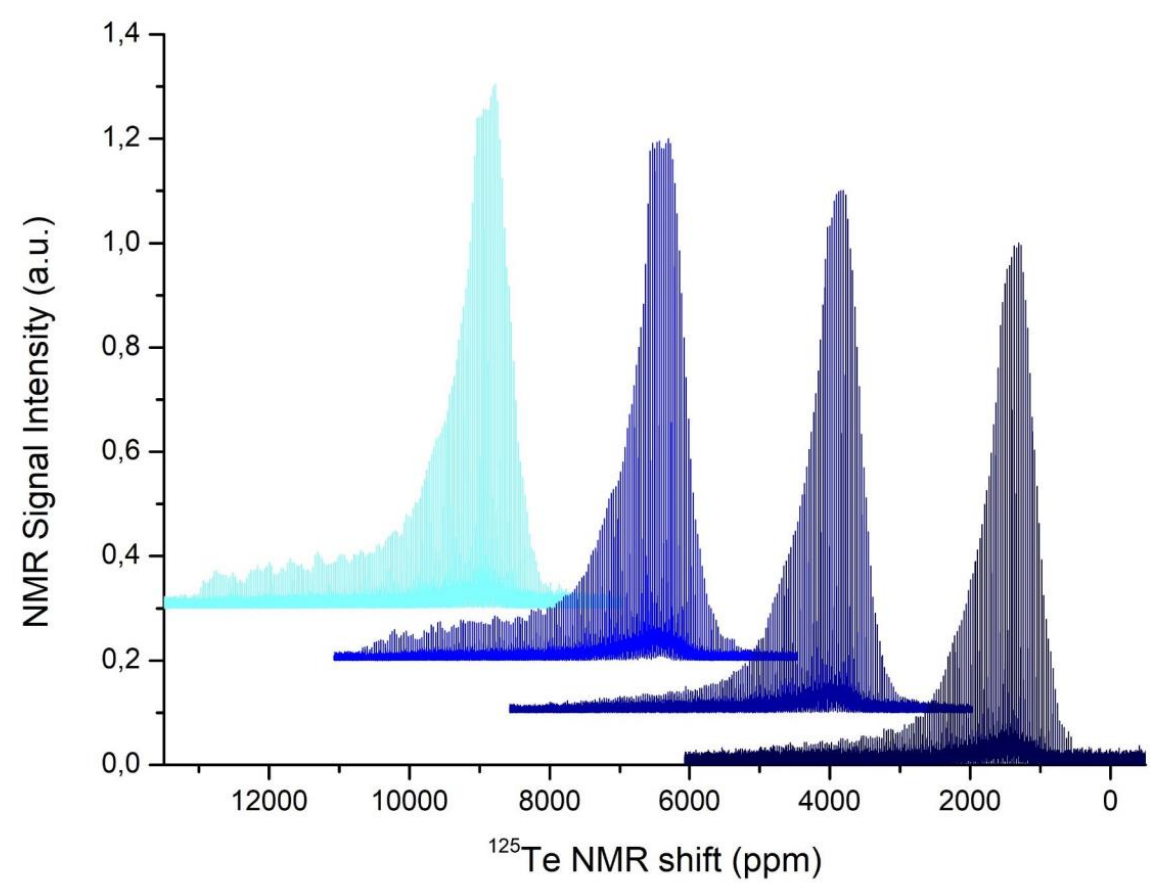


Figure 10

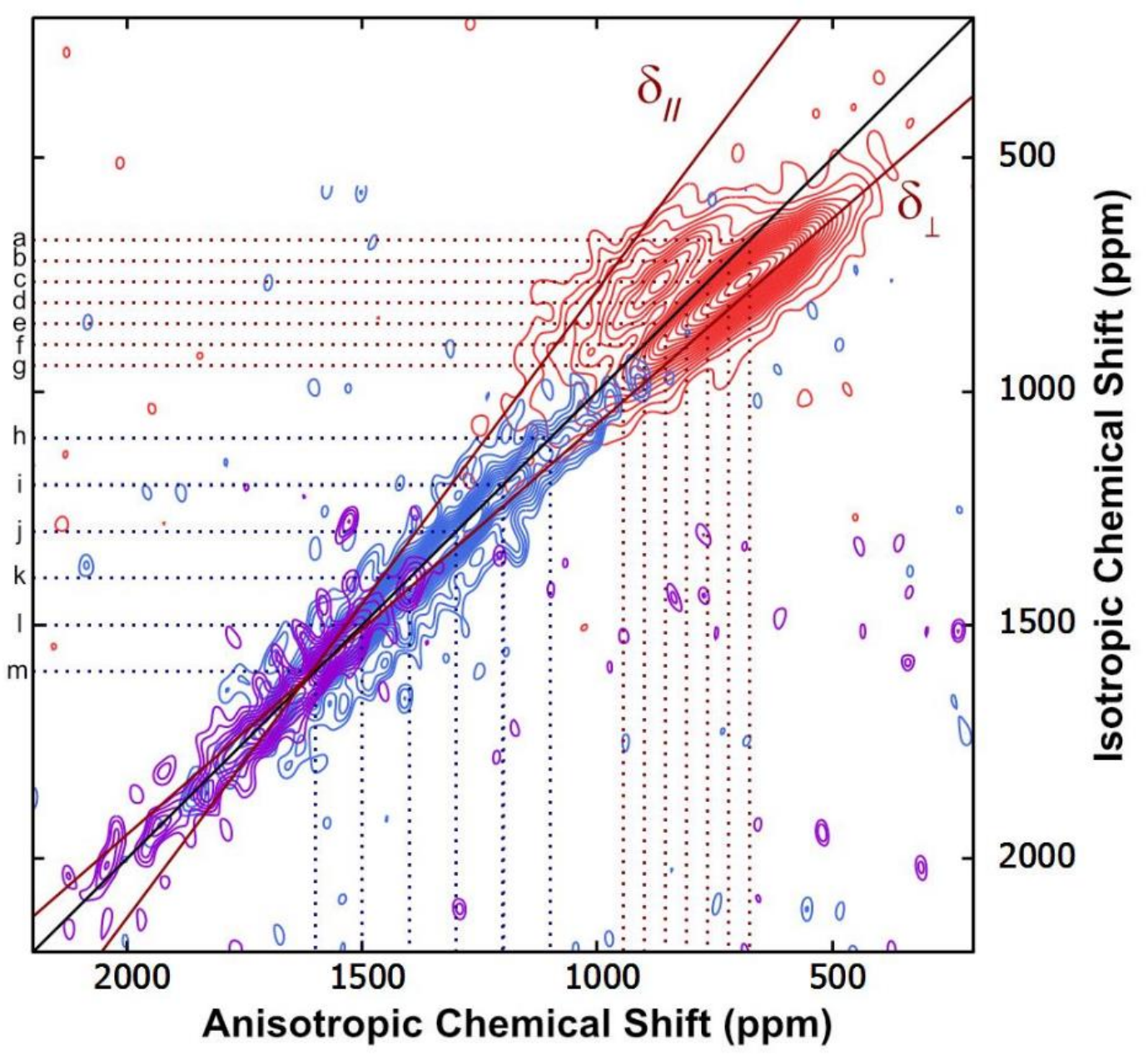


Figure 11

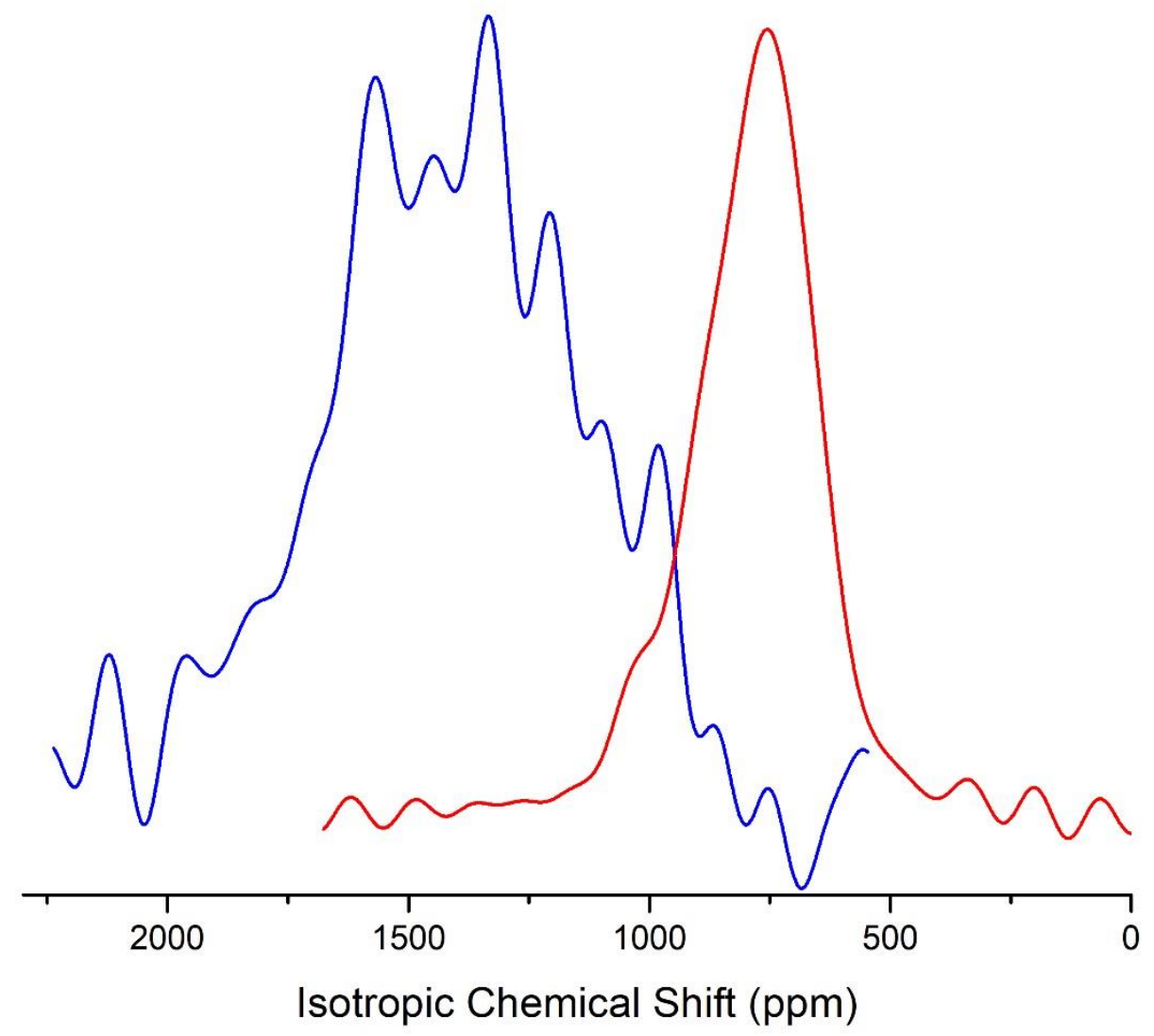


Figure 12

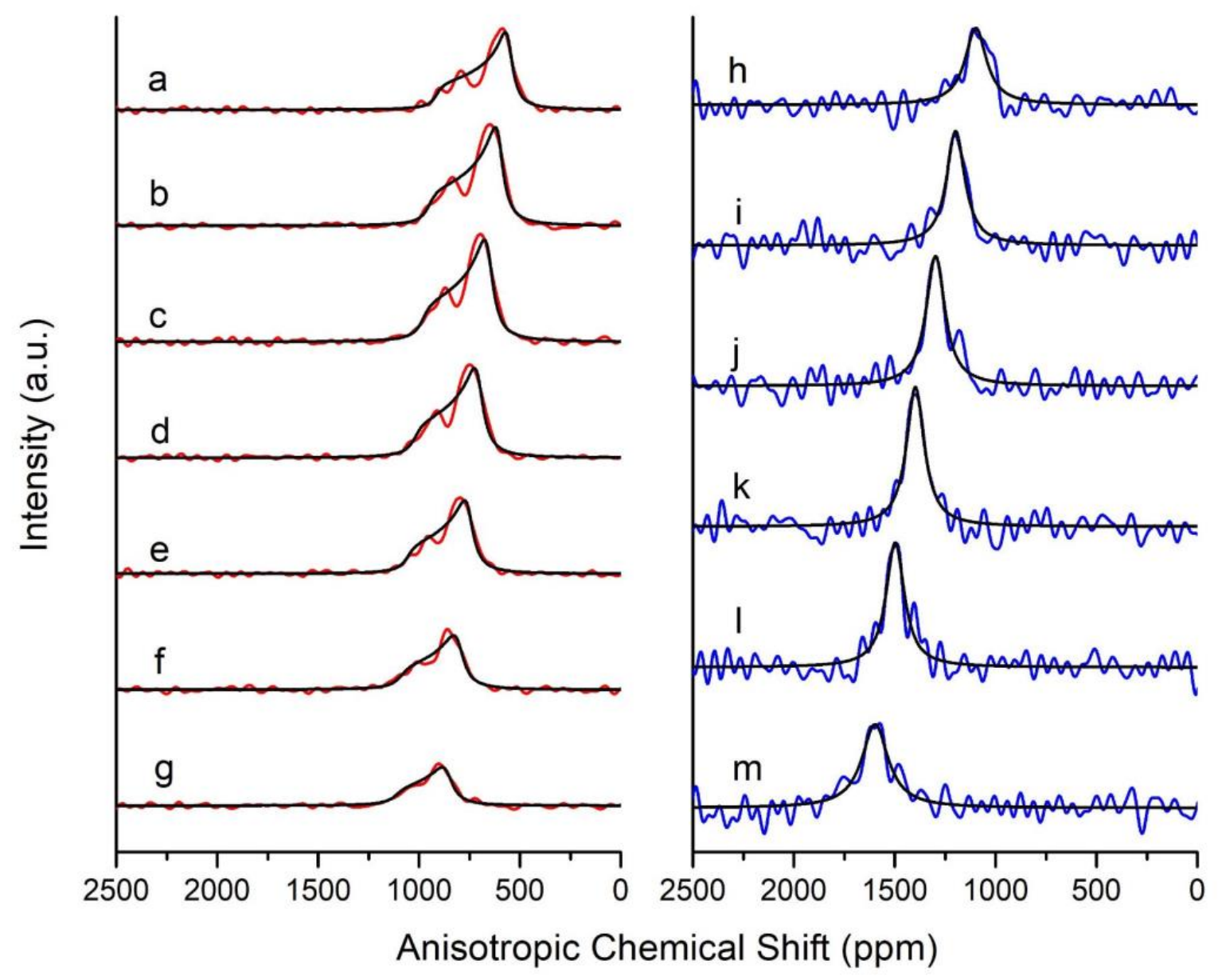


Figure 13

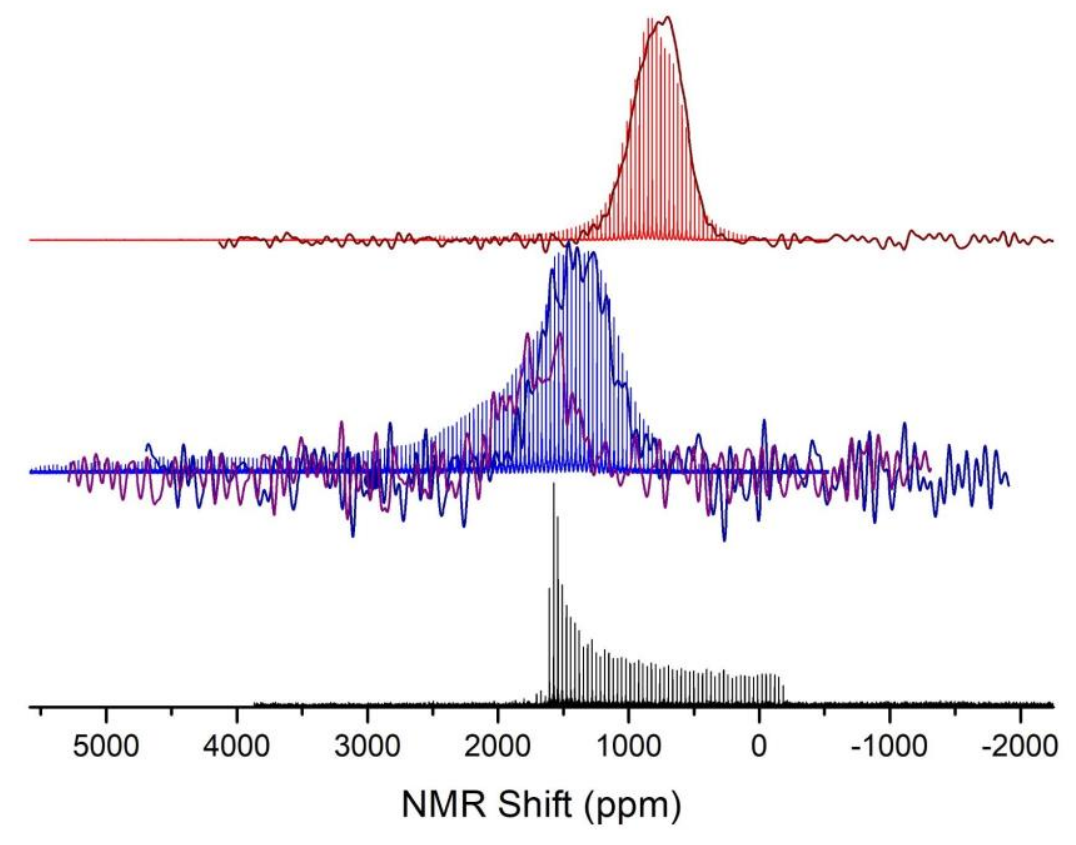




\section{References}

1. W. Zhang, R. Mazzarello, M. Wuttig, E. Ma, « Designing crystallization in phase-change materials for universal memory and neuro-inspired computing », Nature Reviews Materials 4 (2019) 150 (DOI: doi.org/10.1038/s41578-018-0076-x)

2 M. Wuttig, "Towards a universal memory” Nat. Mater. 4, 265-266 (2005)

3 M. Wuttig, N. Yamada, "Phase-change materials for rewriteable data storage. Nat. Mater. 6, 824-832 (2007)

4. D. Lencer, M. Salinga, B. Grabowski, T. Hickel, J. Neugebauer, M. Wuttig, « A map for phase-change materials », Nature Mater. 7 (2008) 972 (DOI : doi.org/10.1038/nmat2330)

5. J.-Y. Raty, M. Schumacher, P. Golub, V.L. Deringer, C. Gatti, M. Wuttig, « A Quantum-Mechanical Map for Bonding and Properties in Solids », Adv. Mater 31 (2019) 1806280 (DOI :

doi.org/10.1002/adma.201806280)

6. Simone Raoux, Matthias Wuttig (Eds.), «Phase Change Materials: Science and Applications », Springer Verlag, New York (2009) p. 141.

7. Alexander V. Kolobov, Junji Tominaga (Eds.), « Chalcogenides: Metastability and Phase Change Phenomena », Springer, Berlin (2012)

8. Redaelli, A. (Ed.), « Phase Change Memory: Device Physics, Reliability and Applications »; Springer: Cham, Switzerland, (2018)

9. « N.M.R. Orbital shifts of ${ }^{125} \mathrm{Te}$ in solids,dependance on ionicity and local structure », A. Willig et B. Sapoval, Journal de Physique Lettres (1977) 38, 57-60 (doi.org/10.1051/jphyslet:0197700380205700)

10. « Multinuclear Solid-State NMR of Inorganic Materials », K. J.D. MacKenzie, M. E. Smith, PERGAMON 2002

11. « ${ }^{125}$ Te NMR study of tellurite crystals », S. Sakida, S. Hayakawa, T. Yoko, Journal of Non-Crystalline Solids (1999) 243 1-12 (doi.org/10.1016/S0022-3093(98)00811-4) 
12. « Solid-state tellurium-125 nuclear magnetic resonance studies of transition-metal ditellurides », I. Orion, J. Rocha, S. Jobic, V. Abadie, R. Brec, C. Fernandez, J.-P. Amoureux, J. Chem. Soc., Dalton Trans. (1997) 3741-3748 (doi: 10.1039/A704757F)

13. D.C. Bobela, P. Taylor, « Nuclear magnetic Resonance studies of Tellurium and Antimony Bonding in Crystal $\mathrm{Sb}_{2} \mathrm{Te}_{3}, \mathrm{GeTe}$, and $\mathrm{Ge}_{2} \mathrm{Sb}_{2} \mathrm{Te}_{5}$ », Jpn. J. Appl. Phys. 47 (2008) 8162 (DOI: doi.org/10.1143/JJAP.47.8162)

14. D.C. Bobela, P. Taylor, "Tellurium bonding in amorphous $\mathrm{Ge}_{2} \mathrm{Sb}_{2} \mathrm{Te}_{4}, \mathrm{Ge}_{2} \mathrm{Sb}_{2} \mathrm{Te}_{5}$ and $\mathrm{Ge}_{2} \mathrm{Sb}_{2} \mathrm{Te}_{7}: \mathrm{A}$ 125Te NMR investigation », J. Non-Cryst. Solids 354 (2008) 2665-2668 (DOI : doi.org/10.1016/j.jnoncrysol.2007.09.052)

15. T.G. Edwards, E.L. Gjersing, S. Sen, S.C. Currie, B.G. Aitken, “ ${ }^{125}$ Te NMR chemical shifts and tellurium coordination environments in crystals and glasses in the Ge-As-Sb-Te sytem », J. Non-Crys. Solids 357 (2011) 3036-3041 (DOI : doi.org/10.1016/j.jnoncrysol.2011.04.007)

16. D. Massiot, I.Farnan, N. Gautier, D. Trumeau, A. Trokiner, J.-P. Coutures, « 71Ga and 69Ga nuclear magnetic resonance study of $\mathrm{P}-\mathrm{Ga}, \mathrm{O}$,:resolution of four- and six-fold coordinated Ga sites in static conditions », Solid State NMR 4 (1995) 241-248 (DOI:doi.org/10.1016/0926-2040(95)00002-8)

17. S. Sen, T.G. Edwards, J.-Y. Cho, Y.-C. Joo, « Te-Centric View of the Phase Change Mechanism in GeSb-Te Alloys », Physical Review Letters 108 (2012) 195506, (DOI: 10.1103/PhysRevLett.108.195506)

18. E.M. Levin, M.F. Besser, R. Hanus « Electronic and thermal transport in GeTe : a versatile base for thermoelectric materials », J. Appl. Phys. 114 (2013) 083713 (DOI : doi.org/10.1063/1.4819222)

19. E.M. Levin, « Effects of Ge substitution in GeTe by Ag or Sb on the Seebeck coefficient and carrier concentration derived from ${ }^{125}$ Te NMR »,Phys. Rev. B 93 (2016) 045209 (DOI : doi.org/10.1103/PhysRevB.93.045209)

20. E.M. Levin, « Charge carrier effective mass and conncentration derived form combination of Seebeck coefficient and ${ }^{125}$ Te NMR measurements in complex tellurides », Phys. Rev. B 93 (2016) 245202, (DOI : doi.org/10.1103/PhysRevB.93.245202) 
21. E.M. levin, J.-F. Cui, K. Schmidt-Rohr, « Sub-millisecond ${ }^{125}$ Te NMR spin-lattice relaxation times and large Knight shifts in complex tellurides : Validation of a quadratic relation across the spectrum », Solid-State NMR 78 (2016), 40-44 (DOI : doi.org/10.1016/j.ssnmr.2016.07.003)

22. J. Cui, E.M. Levin, Y. Lee, Y. Furukawa, «Electronic properties of GeTe and Ag-or Sb- substituted GeTe studied by low-temperature ${ }^{125}$ Te NMR », Phys. Rev. B 94 (2016) 085203 (DOI : doi.org/10.1103/PhysRevB.94.085203)

23. E.M. Levin, T.M. Riedmann, A. Howard, N.H. Jo, S.L. Bud'ko, P.C. Canfield, T.A. Lograsso, " ${ }^{125} T e$ NMR and Seebeck Effect in Bi $i_{2} \mathrm{Te}_{3}$ Synthesized from Stoichiometric and Te-Rich Melts », J. Phys. Chem. C. 120 (2016) 25196-25202 (DOI : doi.org/10.1021/acs.jpcc.6b06973)

24. E.M. Levin, C. Cooling, S.L. Bud'ko, W.E. Straszheim, T.A. Lograsso, « Crystal structure, magnetization, ${ }^{125} \mathrm{Te}$ NMR and Seebeck coefficient of $G e_{49} \operatorname{Te}_{50} R_{1}(R=l a, P r, G d, D y$ and $Y b)$ », J. Mat. Chem. Phys. 192 (2017) 58-66 (DOI : doi.org/10.1016/j.matchemphys.2017.01.038)

25. B. Njegic, E.M. Levin, K. Schmidt-Rohr, " ${ }^{125}$ Te NMR Chemical-Shift trends in PbTe-GeTe and PbTeSnTe alloys », Solid State Nuclear Magnetic Resonance 55-56 (2013) 79-83 (DOI : doi.org/10.1016/j.ssnmr.2013.09.003)

26. M.N. Garaga, U. Werner-Zwanziger, J. W. Zwanziger, “ ${ }^{125}$ Te NMR Probes of Tellurium Oxide Crystals : Shielding-Structure Correlations », Inorg. Chem. 57 (2017) 892-898 (DOI : doi.org/10.1021/acs.inorgchem.7b02913)

27. L. A. O’Dell, R. W. Schurko, «QCPMG using adiabatic pulses for faster acquisition of ultra-wideline NMR Spectra », Chemical Physics Letters 464 (2008) 97-102 (DOI : doi.org/10.1016/j.cplett.2008.08.095)

28. A. W. MacGregor, L. A. O’Dell, R. W. Schurko, "New methods for the acquisition of ultra-wideline solid-state NMR spectraof spin-1/2 nuclides”, Journal of Magnetic Resonance 208 (2011) 103-113 doi.org/10.1016/j.jmr.2010.10.011

29. D.C. Kaseman, I. Hung, K. Lee, K. Kovnir, Z. Gen, B. Aitken, S. Sen, « Tellurium Speciation, Connectivity, and Chemical Order in As Te $_{100-x}$ Glasses : Results from Two-Dimensional ${ }^{125}$ Te NMR Spectroscopy », J. Phys. Chem. B (2015), (DOI: føi.org/10.1021/jp5123618) 
30. Z. Whittles, M. Marple, I. Hung, Z. Gan, S. Sen, " Structure of BaO-TeO $\mathrm{B}_{2}$ glasses : A two-dimensional ${ }^{125}$ Te NMR spectroscopic study », J. Non-Cryst. Solids 481 (2018) 282-288 (DOI : doi.org/10.1016/j.jnoncrysol.2017.10.055)

31. M.A.T. Marple, M. Jesuit, I. Hung, Z. Gan, S. Feller, S. Sen, « Structure of TeO $\mathrm{O}_{2}$ glass : Results from $2{ }^{125}$ Te NMR spectroscopy », J. Non-Cryst. Solids 513 (2019) 183-190 (DOI : doi.org/10.1016/j.jnoncrysol.2019.03.019)

32. D. H. Damon, M. S. Lubell, R. Mazelsky, "Nature of the defects in germanium Telluride", J. Phys. Chem. Solids 28, 520-522 (1966) doi 10.1016/0022-3697(67)90323-X

33. J. E. Lewis, « Band Structure and Nature of Lattice Defects in GeTe from Analysis of Electrical Properties", phys. stat. sol. 36, 737 (1969) doi.org/10.1002/pssb.19690350224 ”, phys. stat. sol. 38, 131 (1970) 34. J. E. Lewis, "The Defect Structure of Non-Stoichiometric Germanium Telluride from lnagnetic Susceptibility Measurements”, phys. stat. sol. 38, 131 (1970), doi.org/10.1002/pssb.19700380110 35. A. H Edwards, A. C Pineda, P. A Schultz, M. G Martin, A. P Thompson, H. P Hjalmarson, “Theory of persistent, p-type, metallic conduction in c-GeTe”, J. Phys.: Condens. Matter 17 (2005) L329-L335, doi:10.1088/0953-8984/17/32/L01

36. A H. Edwards, A. C. Pineda "Electronic structure of intrinsic defects in crystalline germanium telluride”, PRB 73, 045210 (2006), DOI: 10.1103/PhysRevB.73.045210

37. I. Hung, Z. Gan, " On the practical aspects of recording wideline QCPMG NMR Spectra », Journal of Magnetic Resonance 204 (2010) 256-265 (DOI : doi.org/10.1016/j.jmr.2010.03.001)

38. P. Blaha, K.Schwarz, F. Tran, R. Laskowski, G.K.H. Madsen and L.D. Marks, « WIEN2k: An APW+lo program for calculating the properties of solids. ", J. Chem. Phys. 152, 074101 (2020) doi.org/10.1063/1.5143061

39. L.E. Shelimova, O.G. Karpinskii, V.S. Zemskov, P.P. Konstantinov, « Structural and Electrical Properties of Layered Tetradymite-like Compounds in the GeTe-Bi2Te3 and GeTe-Sb2Te3 Systems », Inorg. Mater. (2000) 36, 235-242 DOI: 10.1007/BF02757928

40. L.E. Shelimova, O.G. Karpinskii, E.S. Avilov, M.A. Kretova, «Physicochemical investigation of solid solutions based on germanium telluride in the Ge-Te-5ba system », Inorg. Mater. (1991) 27, 1950-1954 
41. D. I. Bletskan, "Phase equilibrium in the systems $\mathrm{A}^{\mathrm{IV}}-\mathrm{B}^{\mathrm{VI}}-$ Part. II: Systems Germanium-Chalcogen", Journal of Ovonic Research Vol. 1, No. 5, October 2005, p. 53 - 60

42. A.V. Novoselova, V.P. Zlomanov, S. G. Karbanov, O. V. Matveyev, A. M. Gas’kov, « Physico-Chemical Study of the Germanium, Tin, Lead, Chalcogenids, Progress in Solid State Chemistry (1972), 7, 85115

43. D. Massiot, F. Fayon, M. Capron, I. King, S. Le Calvé, B. Alonso, J-O. Durand, B. Bujoli, Z. Gan, G. Hoatson, « Modelling one- and two-dimensional Solid State NMR spectra. », Magnetic Resonance in Chemistry, 40 70-76 (2002) 